\title{
WANDERING VECTORS AND THE REFLEXIVITY OF FREE SEMIGROUP ALGEBRAS
}

\author{
MATTHEW KENNEDY
}

\begin{abstract}
A bStract. A free semigroup algebra $\mathcal{S}$ is the weak-operator-closed (non-selfadjoint) operator algebra generated by $n$ isometries with pairwise orthogonal ranges. A unit vector $x$ is said to be wandering for $\mathcal{S}$ if the set of images of $x$ under non-commuting words in the generators of $\mathcal{S}$ is orthonormal.

We establish the following dichotomy: either a free semigroup algebra has a wandering vector, or it is a von Neumann algebra. Consequences include that every free semigroup algebra is reflexive, and that certain free semigroup algebras are hyper-reflexive with a very small hyper-reflexivity constant.
\end{abstract}

\section{Introduction}

A free semigroup algebra $\mathcal{S}$ is the weak-operator-closed (non-self-adjoint) algebra generated by $n$ isometries $S_{1}, \ldots, S_{n}$ on a Hilbert space $\mathcal{H}$ which have pairwise orthogonal ranges, or equivalently, which satisfy

$$
S_{i}^{*} S_{j}= \begin{cases}I & \text { if } i=j, \\ 0 & \text { otherwise. }\end{cases}
$$

Although $n$ can be finite or infinite, for notational convenience we treat $n$ as finite and make note of any issues that arise. We say that $S=\left[\begin{array}{lll}S_{1} & \ldots & S_{n}\end{array}\right]$ is a row isometry, since $S$ is isometric as a row operator from $\mathcal{H}^{n}$ to $\mathcal{H}$.

Row isometries arise throughout operator theory. A theorem of Frazho, Bunce, and Popescu shows that $n$ operators $A_{1}, \ldots, A_{n}$ which satisfy $\sum A_{k} A_{k}^{*} \leq I$ can be dilated to a row isometry $S=\left[\begin{array}{lll}S_{1} & \ldots & S_{n}\end{array}\right]$ such that

$$
S_{k}=\left(\begin{array}{cc}
A_{k} & 0 \\
* & *
\end{array}\right)
$$

This is a non-commutative multivariable analogue of the Sz.-Nagy dilation theorem.

Popescu [19] showed that the norm-closed algebra generated by any row isometry of size $n$ is completely isometrically isomorphic to the non-commutative disk algebra $\mathcal{A}_{n}$, and it is well known that the $\mathrm{C}^{*}$-algebra generated by a row isometry of size $n$ is isomorphic to the Cuntz algebra $\mathcal{O}_{n}$ if $\sum A_{k} A_{k}^{*}=I$, and otherwise is isomorphic to the Cuntz-Toeplitz algebra $\mathcal{E}_{n}$. By contrast, the weak-operator-closed algebras generated by distinct row isometries can be dramatically different (see for example [10]).

In some sense then, it is natural to study row isometries by looking at the free semigroup algebras they generate. This idea, and with it the definition of a free semigroup algebra, was introduced by Davidson and Pitts [12]. They observed that

2000 Mathematics Subject Classification. Primary 47L80; Secondary 47L55, 47A15.

Research supported by NSERC. 
free semigroup algebras often contain interesting information about the unitary invariants of their generators.

The prototypical example of a free semigroup algebra is the non-commutative analytic Toeplitz algebra generated by the left regular representation of the free semigroup on $n$ letters. This algebra, which we denote by $\mathcal{L}_{n}$, was first studied by Popescu [18] in the context of non-commutative multivariable dilation theory.

For $n=1, \mathcal{L}_{n}$ is the familiar algebra of analytic Toeplitz operators, which is singly generated by the unilateral shift. For $n \geq 2, \mathcal{L}_{n}$ is no longer commutative, but it turns out that a number of classical results about the analytic Toeplitz operators have straightforward generalizations to this setting. This is a large part of the motivation for the name "non-commutative analytic Toeplitz algebra."

The role of $\mathcal{L}_{n}$ is of central importance in the general theory of free semigroup algebras, and it turns out to be desirable to isolate " $\mathcal{L}_{n}$-like" behavior. A free semigroup algebra is said to be of type $L$ if it is algebraically isomorphic to $\mathcal{L}_{n}$. It is important to emphasize the word "algebraically" here. Examples have been constructed (see for example [10]) of free semigroup algebras which are of type $\mathrm{L}$, and so behave algebraically like $\mathcal{L}_{n}$, but which have a very different spatial structure.

The general structure theorem for free semigroup algebras [10] shows that every free semigroup algebra can be decomposed into $2 \times 2$ block-lower-triangular form, where the left column is a slice of a von Neumann algebra, and the bottom-right entry is a type L free semigroup algebra. It is well known (see for example 22]) that the weak-operator-closed algebra generated by a single isometry can be selfadjoint. Davidson, Katsoulis, and Pitts [10] asked whether it was possible for a free semigroup algebra on 2 or more generators to be self-adjoint, and some time later Read [21] (see also [9]) answered in the affirmative by showing that $\mathcal{B}(\mathcal{H})$ was a free semigroup algebra.

A notion of fundamental importance is that of a wandering vector. A unit vector $x$ is said to be wandering for a free semigroup algebra $\mathcal{S}$ if the set of images of $x$ under non-commuting words in the generators of $\mathcal{S}$ forms an orthonormal set. It is known (see for example [12]) that the spatial structure of $\mathcal{L}_{n}$ is completely determined by the existence of a large number of wandering vectors.

It is easy to see that the restriction of any free semigroup algebra to the cyclic subspace generated by a wandering vector is unitarily equivalent to $\mathcal{L}_{n}$, and so in particular is of type L. It has been an open question for some time, however, whether every type L free semigroup algebra necessarily has a wandering vector. It turns out that this question is equivalent to the question of whether every free semigroup algebra is reflexive. This can be shown using the general structure theorem for free semigroup algebras: since every von Neumann algebra is reflexive, the reflexivity of a free semigroup algebra depends on the reflexivity of its type L part.

The purpose of this paper is to prove that every type L free semigroup algebra has wandering vectors, and hence to prove that every free semigroup algebra is reflexive.

Our approach is very much in the spirit of the "dual algebra arguments" which have been used with great success by Bercovici, Foias, Pearcy and many others (see for example [5]), and which are based on Brown's proof of the existence of invariant subspaces for subnormal operators [6]. The fundamental idea at the heart of these arguments is that it is often possible to prove the existence of invariant subspaces 
for a weak*-closed operator algebra by showing that, in an appropriate sense, the predual of the algebra is small.

Typically, these arguments are employed in a commutative setting, where certain spectral and function-theoretic tools are available. In the present non-commutative context, we rely instead on various operator-theoretic techniques.

A clue that it might be possible to attack the present problem using dual algebra techniques came from a recent paper of Bercovici [4], who used them to establish the hyper-reflexivity of a class of algebras which includes the non-commutative analytic Toeplitz algebra on two or more generators. The hyper-reflexivity of this algebra had already been shown by Davidson and Pitts [12, with an upper bound of 51 on the hyper-reflexivity constant, but Bercovici's approach yielded a surprisingly low upper bound of 3 .

Motivated by Bercovici's result, once we have shown that every type L free semigroup algebra has a wandering vector, we go further and show that every type $\mathrm{L}$ free semigroup algebra on two or more generators is hyper-reflexive with hyperreflexivity constant at most 3 .

\section{Preliminaries}

Let $\mathbb{F}_{n}^{+}$denote the free semigroup in $n$ non-commuting letters $\{1, \ldots, n\}$, including the empty word $\varnothing$. For a word $w$ in $\mathbb{F}_{n}^{+}$, let $|w|$ denote its length, and let $\mathbb{F}_{n}^{k}$ denote the set of all words in $\mathbb{F}_{n}^{+}$of length at most $k$.

Let $\mathcal{F}_{n}$ denote the "Fock" space $\mathcal{F}_{n}=\ell^{2}\left(\mathbb{F}_{n}^{+}\right)$with orthonormal basis $\left\{\xi_{w}: w \in\right.$ $\left.\mathbb{F}_{n}^{+}\right\}$consisting of words in $\mathbb{F}_{n}^{+}$. For each $v$ in $\mathbb{F}_{n}^{+}$, define an isometry $L_{v}$ by

$$
L_{v} \xi_{w}=\xi_{v w}, \quad w \in \mathbb{F}_{n}^{+} .
$$

The map $v \rightarrow L_{v}$ gives a representation of $\mathbb{F}_{n}^{+}$, called the left regular representation.

The isometries $L_{1}, \ldots, L_{n}$ have pairwise orthogonal ranges. The free semigroup algebra they generate, denoted by $\mathcal{L}_{n}$, is called the non-commutative analytic Toeplitz algebra. For $n=1, \mathcal{L}_{n}$ is the classical analytic Toeplitz algebra, but for $n \geq 2, \mathcal{L}_{n}$ is no longer commutative.

We require a result for $\mathcal{L}_{n}$ which generalizes a classical result about the analytic Toeplitz operators. An element in $\mathcal{L}_{n}$ is said to be inner if it is an isometry, and outer if it has dense range. It was shown in [12] that an arbitrary element $A$ in $\mathcal{L}_{n}$ can be written as $A=B C$, where $B$ is inner and $C$ is outer. This generalizes the classical inner-outer factorization for elements in the analytic Toeplitz algebra.

Every element $A$ in $\mathcal{L}_{n}$ is completely determined by its Fourier series

$$
A \sim \sum_{w \in \mathbb{F}_{n}^{+}} a_{w} L_{w}
$$

which is a formal power series with coefficients in $\mathcal{L}_{n}$, where

$$
A \xi_{\varnothing}=\sum_{w \in \mathbb{F}_{n}^{+}} a_{w} \xi_{w} .
$$

For $k \geq 1$, define the $k$-th Cesaro sum of the Fourier series of $A$ by

$$
\Gamma_{k}(A)=\sum_{|w|<k}\left(1-\frac{|w|}{k}\right) a_{w} L_{w} .
$$

Then the sequence $\Gamma_{k}(A)$ is strongly convergent to $A$. 
By symmetry, for each $v$ in $\mathbb{F}_{n}^{+}$we can define an isometry $R_{v}$ by

$$
R_{v} \xi_{w}=\xi_{w v}, \quad w \in \mathbb{F}_{n}^{+},
$$

and the map $v \rightarrow R_{v}$ gives an anti-representation (i.e. a multiplication reversing representation) of $\mathbb{F}_{n}^{+}$, called the right regular representation. The isometries $R_{1}, \ldots, R_{n}$ also have orthogonal ranges, and the free semigroup algebra they generate, denoted by $\mathcal{R}_{n}$, is unitarily equivalent to $\mathcal{L}_{n}$. It was shown in [12] that $\mathcal{R}_{n}$ is the commutant of $\mathcal{L}_{n}$.

A free semigroup algebra $\mathcal{S}$ is said to be of type $L$ if it is algebraically isomorphic to $\mathcal{L}_{n}$. It was shown in [10] that if $\mathcal{S}$ is of type $\mathrm{L}$, then there is a completely isometric isomorphism $\Phi$ from $\mathcal{L}_{n}$ to $\mathcal{S}$ which takes the generators of $\mathcal{L}_{n}$ to the generators of $\mathcal{S}$. Moreover, $\Phi$ is a weak*-to-weak* homeomorphism, and the inverse map $\Phi^{-1}$ is the dual of an isometric isomorphism $\phi$ from the predual of $\mathcal{L}_{n}$ to the predual of $\mathcal{S}$.

For a free semigroup algebra $\mathcal{S}$, let $\mathcal{S}_{0}$ denote the weak-operator-closed ideal generated by $S_{1}, \ldots, S_{n}$. Then either $\mathcal{S}_{0}=\mathcal{S}$, or $\mathcal{S} / \mathcal{S}_{0} \cong \mathbb{C}$. In the latter case, the general structure theorem for free semigroup algebras [10] implies that $\mathcal{S}$ has a type L part. If $\mathcal{S}_{0}=\mathcal{S}$, then $\mathcal{S}$ is a von Neumann algebra.

The set of weak*-continuous linear functionals on $\mathcal{B}(\mathcal{H})$, i.e. the predual, can be identified with the set of trace class operators $\mathcal{C}^{1}(\mathcal{H})$, where $K$ in $\mathcal{C}^{1}(\mathcal{H})$ corresponds to the linear functional

$$
T \rightarrow \operatorname{tr}(T K), \quad T \in \mathcal{B}(\mathcal{H}) .
$$

With this identification, the set of weak-operator-continuous linear functionals on $\mathcal{B}(\mathcal{H})$ corresponds to the set of finite rank operators. The predual of a weak*-closed subspace $\mathcal{S}$ of $\mathcal{B}(\mathcal{H})$ can be identified with the quotient space $\mathcal{C}^{1}(\mathcal{H}) /^{\perp} \mathcal{S}$, where ${ }^{\perp} \mathcal{S}$ denotes the set of elements in $\mathcal{C}^{1}(\mathcal{H})$ which annihilate $\mathcal{S}$, i.e. the preannihilator.

It was shown in [12] that the weak* topology and the weak operator topology coincide on $\mathcal{L}_{n}$. This means that the coset of every weak*-continuous linear functional on $\mathcal{L}_{n}$ contains an element of finite rank.

Let $\mathcal{S}$ be a free semigroup algebra on a Hilbert space $\mathcal{H}$. A unit vector $x$ in $\mathcal{H}$ is said to be wandering for $\mathcal{S}$ if the set $\left\{S_{w} x: w \in \mathbb{F}_{n}^{+}\right\}$is orthonormal. The following theorem from [10] is integral to our results.

Theorem 2.1. Let $\mathcal{S}$ be a type $L$ free semigroup algebra. Then for some $m \geq 1$, the ampliation $\mathcal{S}^{(m)}$ has a wandering vector.

Suppose that $\mathcal{S}$ is a type L free semigroup algebra, and let $\pi_{0}$ be the weakoperator-continuous linear functional on $\mathcal{S}$ such that $\pi_{0}$ annihilates $\mathcal{S}_{0}$ and $\pi_{0}(I)=$ 1. Then the coset in $\mathcal{C}^{1}(\mathcal{H})$ corresponding to $\pi_{0}$ contains an operator of finite rank, say $m \geq 1$. This $m$ corresponds to the $m$ in the statement of Theorem 2.1. Since the restriction of $\mathcal{S}^{(m)}$ to the cyclic subspace generated by a wandering vector is unitarily equivalent to $\mathcal{L}_{n}$, it follows that the weak* topology and the weak operator topology agree on $\mathcal{S}$.

A subspace $\mathcal{S}$ of $\mathcal{B}(\mathcal{H})$ is said to be reflexive if $\mathcal{S}$ contains every operator $T$ in $\mathcal{B}(\mathcal{H})$ with the property that $T x$ belongs to $\mathcal{S}[x]$ for every $x$ in $\mathcal{H}$. This definition of reflexivity was introduced by Loginov and Shulman [16].

The notion of hyper-reflexivity, which was introduced by Arveson [1], is a quantitative analogue of reflexivity. Let $d_{\mathcal{S}}$ denote the distance seminorm

$$
d_{\mathcal{S}}(T)=\inf \{\|T-A\|: A \in \mathcal{S}\}, \quad T \in \mathcal{B}(\mathcal{H}),
$$


and define another seminorm $r_{\mathcal{S}}$ by

$$
r_{\mathcal{S}}(T)=\sup \{|(T x, y)|:\|x\|,\|y\| \leq 1 \text { and }(A x, y)=0 \text { for all } A \in \mathcal{S}\}, \quad T \in \mathcal{B}(\mathcal{H}) .
$$

Then the reflexivity of $\mathcal{S}$ is equivalent to the condition that $d_{\mathcal{S}}(T)=0$ if and only if $r_{\mathcal{S}}(T)=0$.

The equality $r_{\mathcal{S}}(T) \leq d_{\mathcal{S}}(T)$ always holds. We say that $\mathcal{S}$ is hyper-reflexive if there is a constant $C>0$ such that $d_{\mathcal{S}}(T) \leq C r_{\mathcal{S}}(T)$ for all $T$ in $\mathcal{B}(\mathcal{H})$. The smallest such $C$ is called the hyper-reflexivity constant of $\mathcal{S}$. Of course, hyperreflexivity implies reflexivity.

Davidson [8] showed that the analytic Toeplitz algebra is hyper-reflexive with hyper-reflexivity constant at most 19. Davidson and Pitts [12] showed that for $n \geq 2, \mathcal{L}_{n}$ is hyper-reflexive with hyper-reflexivity constant at most 51 . This was later improved by Bercovici [4], who showed that this hyper-reflexivity constant is at most 3 .

\section{The Non-commutative Toeplitz Operators}

The Toeplitz operators are precisely the operators $T$ in $\mathcal{B}\left(\ell^{2}(\mathbb{N})\right)$ which satisfy $S^{*} T S=T$, where $S$ is the unilateral shift. This motivates the following definition, which was introduced by Popescu [17].

Definition 3.1. Let $\mathrm{S}=\left[\begin{array}{llll}S_{1} & \ldots & S_{n}\end{array}\right]$ be a row isometry. We say that $T$ is an $S$ Toeplitz operator if

$$
S_{i}^{*} T S_{j}= \begin{cases}T & \text { if } i=j \\ 0 & \text { otherwise }\end{cases}
$$

and we let $\mathcal{T}_{S}$ denote the set of all $S$-Toeplitz operators.

If an $S$-Toeplitz operator $T$ is strictly positive, then by Theorem 4.3 of [17], it can be factored as $T=A^{*} A$, for some $A$ in the commutant of the free semigroup algebra generated by $\mathcal{S}$.

Define row isometries $L$ and $R$ by $L=\left[\begin{array}{lll}L_{1} & \ldots & L_{n}\end{array}\right]$ and $R=\left[\begin{array}{lll}R_{1} & \ldots & R_{n}\end{array}\right]$. The size, $n$, will always be clear from the context. In this section we will establish some properties of the set $\mathcal{T}_{R}$ of $R$-Toeplitz operators which we will need later. Note that since $\mathcal{L}_{n}$ is unitarily equivalent to $\mathcal{R}_{n}$, the set $\mathcal{T}_{R}$ of $R$-Toeplitz operators is unitarily equivalent to the set $\mathcal{T}_{L}$ of $L$-Toeplitz operators. This means that any properties of $\mathcal{T}_{R}$ will correspond in an obvious way to properties of $\mathcal{T}_{L}$.

The following Lemma is implied by Corollary 1.3 of [20]. Here we give a short direct proof.

Lemma 3.2. The set $\mathcal{T}_{R}$ of $R$-Toeplitz operators is precisely the weak* closure of the operator system $\mathcal{L}_{n}^{*}+\mathcal{L}_{n}$.

Proof. It is clear that the weak* closure of $\mathcal{L}_{n}^{*}+\mathcal{L}_{n}$ is contained in $\mathcal{T}_{R}$, since

$$
R_{i}^{*} L_{w} R_{j}=R_{i}^{*} R_{j} L_{w}= \begin{cases}L_{w} & \text { if } i=j, \\ 0 & \text { otherwise. }\end{cases}
$$

Suppose then that $T$ belongs to $\mathcal{T}_{R}$. It's clear that $T^{*}$ also belongs to $\mathcal{T}_{R}$, and hence that the real and imaginary parts of $T$ belong to $\mathcal{T}_{R}$. Since the scalar operators also belong to $\mathcal{T}_{R}$, it follows that we can write $T$ as a finite linear combination of strictly positive operators in $\mathcal{T}_{R}$. Hence we may suppose that $T$ is strictly positive. 
By Theorem 4.3 of [17], we can write $T=A^{*} A$ for some $A$ in $\mathcal{L}_{n}$. Note that $A^{*} \Gamma_{k}(A)$ belongs to $\mathcal{L}_{n}+\mathcal{L}_{n}^{*}$ for $k \geq 1$, where $\Gamma_{k}(A)$ denotes the $k$-th Cesaro sum of the Fourier series for $A$. The sequence $\Gamma_{k}(A)$ is weak*-convergent to $A$, so it follows that $A^{*} \Gamma_{k}(A)$ is weak*-convergent to $A^{*} A=T$, and hence that $T$ belongs to the weak* closure of $\mathcal{L}_{n}+\mathcal{L}_{n}^{*}$.

Note that based on the definition of the set $\mathcal{T}_{R}$ of $R$-Toeplitz operators, Lemma 3.2 implies that the weak ${ }^{*}$ closure of $\mathcal{L}_{n}^{*}+\mathcal{L}_{n}$ is closed in the weak operator topology.

Lemma 3.3. For $n \geq 2$, every $R$-Toeplitz operator $T$ can be factored as $T=B^{*} C$ for some $B$ and $C$ in $\mathcal{L}_{n}$. Moreover, $B$ and $C$ can be taken to be bounded below.

Proof. As in the proof of Lemma 3.2, we can write $T$ as a finite linear combination of strictly positive $R$-Toeplitz operators, say $T=\sum_{i=1}^{m} c_{i} T_{i}$ for some $c_{1}, \ldots, c_{m}$ in $\mathbb{C}$ and strictly positive $T_{1}, \ldots, T_{m}$ in $\mathcal{T}_{R}$. By Theorem 4.3 of [17], we can factor each $T_{i}$ as $T_{i}=A_{i}^{*} A_{i}$ for some $A_{i}$ in $\mathcal{L}_{n}$. Set $B=\sum_{i=1}^{m} L_{1^{i} 2} A_{i}$ and $C=\sum_{i=1}^{m} c_{i} L_{1^{i} 2} A_{i}$. Then $B$ and $C$ both belong to $\mathcal{L}_{n}$ and $T=B^{*} C$.

To see that $B$ and $C$ can be taken to be bounded below, take $B^{\prime}=B+L_{1^{m+1} 2}$ and $C^{\prime}=C+L_{1^{m+2} 2}$. Then $B^{\prime}$ and $C^{\prime}$ both belong to $\mathcal{L}_{n}$. Since the isometries $L_{12}, \ldots, L_{1^{m+22}}$ have pairwise orthogonal ranges, $B^{\prime}$ and $C^{\prime}$ are bounded below, and $T=\left(B^{\prime}\right)^{*} C^{\prime}$.

Lemma 3.3 provides another characterization of the $R$-Toeplitz operators for $n \geq 2$.

Corollary 3.4. For $n \geq 2$, the set $\mathcal{T}_{R}$ of $R$-Toeplitz operators is precisely $\mathcal{L}_{n}^{*} \mathcal{L}_{n}=$ $\left\{B^{*} C: B, C \in \mathcal{L}_{n}\right\}$.

Popescu [20] showed that every $R$-Toeplitz operator $T$ has a Fourier series

$$
T \sim \sum_{w \in \mathbb{F}_{n}^{+}} a_{w} L_{w}+\sum_{w \in \mathbb{F}_{n}^{+} \backslash\{\varnothing\}} \overline{b_{w}} L_{w}^{*},
$$

which is a formal power series with coefficients in $\mathcal{L}_{n}$ and $\mathcal{L}_{n}^{*}$. This completely determines $T$ in the sense that for every word $u$ in $\mathbb{F}_{n}^{+}$,

$$
T \xi_{u}=\sum_{w \in \mathbb{F}_{n}^{+}} a_{w} L_{w} \xi_{u}+\sum_{w \in \mathbb{F}_{n}^{+} \backslash\{\varnothing\}} \overline{b_{w}} L_{w}^{*} \xi_{u} .
$$

Let $\mathcal{S}$ be a type L free semigroup algebra. We know that the canonical map from $\mathcal{L}_{n}$ to $\mathcal{S}$ is a complete isometry and a weak*-to-weak* homeomorphism. Our goal for the remainder of this section is to show that this map extends in a natural way to a map from the weak* closure of $\mathcal{L}_{n}+\mathcal{L}_{n}^{*}$ (i.e. from the set $\mathcal{T}_{R}$ of $R$-Toeplitz operators) to the weak* closure of $\mathcal{S}+\mathcal{S}^{*}$, and that this extension is also a complete isometry and a weak*-to-weak* homeomorphism.

Lemma 3.5. Let $\mathcal{S}$ be a type $L$ free semigroup algebra with $n \geq 2$ generators, and let $\Phi$ be the canonical map from $\mathcal{L}_{n}$ to $\mathcal{S}$. Then $\Phi^{-1}$ maps isometries in $\mathcal{S}$ to isometries in $\mathcal{L}_{n}$.

Proof. By Theorem 2.1, $\mathcal{S}^{(m)}$ has a wandering vector $w$ for some $m$, and the restriction of $\mathcal{S}^{(m)}$ to $\mathcal{S}^{(m)}[w]$ is unitarily equivalent to $\mathcal{L}_{n}$. The map $\Phi^{-1}$ from $\mathcal{S}$ to $\mathcal{L}_{n}$ is given by taking $\mathcal{S}$ to $\mathcal{S}^{(m)}$, restricting to $\mathcal{S}^{(m)}[w]$, and applying this equivalence. If $G$ is an isometry in $\mathcal{S}$, then $G^{(m)}$ is an isometry in $\mathcal{S}^{(m)}$, and so clearly the restriction of $G^{(m)}$ to $\mathcal{S}^{(m)}[w]$ is an isometry. 
Theorem 3.6. Let $\mathcal{S}$ be a type $L$ free semigroup algebra with $n \geq 2$ generators on a Hilbert space $\mathcal{H}$. Then the canonical map $\Phi$ from $\mathcal{L}_{n}$ to $\mathcal{S}$ extends to a completely isometric weak*-to-weak ${ }^{*}$ homeomorphism from the weak ${ }^{*}$ closure of $\mathcal{L}_{n}+\mathcal{L}_{n}^{*}$ to the weak ${ }^{*}$ closure of $\mathcal{S}+\mathcal{S}^{*}$.

Proof. Applying Arveson's extension theorem [2] gives a completely positive map $\Psi$ from $C^{*}\left(\mathcal{L}_{n}\right)$ to $\mathcal{B}(\mathcal{H})$ which extends $\Phi$. Since $\Psi$ extends $\Phi$, we have $\|\Psi\|=$ $\|\Psi(I)\|=\|\Phi(I)\|=1$. Let $\mathcal{Z}=\left\{A \in C^{*}\left(\mathcal{L}_{n}\right): \Psi(A)^{*} \Psi(A)=\Psi\left(A^{*} A\right)\right\}$. By [7], we have

$$
\mathcal{Z}=\left\{A \in C^{*}\left(\mathcal{L}_{n}\right): \Psi(B) \Psi(A)=\Psi(B A) \text { for all } B \text { in } C^{*}\left(\mathcal{L}_{n}\right)\right\} .
$$

By Theorem 4.1 of [10], $\Phi$ maps isometries in $\mathcal{L}_{n}$ to isometries in $\mathcal{S}$, so every isometry in $\mathcal{L}_{n}$ belongs to $\mathcal{Z}$. Since, by Theorem 4.5 of [10], every element in $\mathcal{L}_{n}$ can be written as a finite linear combination of isometries in $\mathcal{L}_{n}$, this implies that $\mathcal{Z}$ contains all of $\mathcal{L}_{n}$. Hence for $A$ in $\mathcal{L}_{n}, \Psi(T A)=\Psi(T) \Psi(A)$ for all $T$ in $C^{*}\left(\mathcal{L}_{n}\right)$. Note that by Corollary 3.4. $C^{*}\left(\mathcal{L}_{n}\right)$ contains $\mathcal{T}_{R}$. For the remainder of the proof, we restrict $\Psi$ to $\mathcal{T}_{R}$.

Let $T$ be a self-adjoint element in $\mathcal{T}_{R}$ such that $\Psi(T)=0$. For sufficiently large $\lambda>0, T+\lambda I$ is strictly positive, so by Theorem 4.3 of [17], we can write $T+\lambda I=B^{*} B$ for some $B$ in $\mathcal{L}_{n}$. Let $V=\lambda^{-1 / 2} B$. Then

$$
\begin{aligned}
\Phi(V)^{*} \Phi(V)-I & =\Psi\left(V^{*} V-I\right) \\
& =\Psi\left(\lambda^{-1} B^{*} B-I\right) \\
& =\Psi\left(\lambda^{-1}(T+\lambda I)-I\right) \\
& =\lambda^{-1} \Psi(T) \\
& =0,
\end{aligned}
$$

which shows that $\Phi(V)$ is an isometry in $\mathcal{S}$. By Lemma 3.5, this implies that $V$ is an isometry in $\mathcal{L}_{n}$. Hence

$$
T=\lambda\left(V^{*} V-I\right)=0
$$

Since, for arbitrary $T$ in $\mathcal{T}_{R}, \operatorname{re}(T)$ and $\operatorname{im}(T)$ are self-adjoint, and since

$$
\Psi(T)=\Psi(\operatorname{re}(T)+\operatorname{im}(T))=\operatorname{re}(\Psi(T))+\operatorname{im}(\Psi(T))=0
$$

if and only if $\psi(\operatorname{re}(T))=0$ and $\psi(\operatorname{im}(T))=0$, it follows that $\Psi$ is injective.

Arguing exactly as above, the canonical map $\Phi^{-1}$ from $\mathcal{S}$ to $\mathcal{L}_{n}$ also has a completely positive extension $\Omega$ from $C^{*}(\mathcal{S})$ to $\mathcal{B}\left(\mathcal{F}_{n}\right)$, and for $G$ in $\mathcal{S}, \Omega(H G)=$ $\Omega(H) \Omega(G)$ for all $H$ in $C^{*}(\mathcal{S})$. Since $\Omega$ extends $\Phi^{-1}$, we have $\|\Omega\|=\|\Omega(I)\|=$ $\left\|\Phi^{-1}(I)\right\|=1$. For the remainder of the proof we restrict $\Omega$ to the intersection of $C^{*}(\mathcal{S})$ and the range of $\Psi$.

Note that the range of $\Psi$ is contained in the weak* closure of $\mathcal{S}+\mathcal{S}^{*}$. Indeed, by Lemma 3.4, every element in the range of $\Psi$ can be written as $\Psi\left(B^{*} C\right)=$ $\Psi\left(B^{*}\right) \Psi(C)=\Phi(B)^{*} \Phi(C)$ for some $B$ and $C$ in $\mathcal{L}_{n}$. The sequence $\Gamma_{k}(C)$ is weak operator convergent to $C$, so by the weak operator continuity of $\Phi$, the sequence $\Phi\left(\Gamma_{k}(C)\right)$ is weak operator convergent to $\Phi(C)$, and hence the sequence $\Phi(B)^{*} \Phi\left(\Gamma_{k}(C)\right)$ is weak operator convergent to $\Phi(B)^{*} \Phi(C)$, which implies that $\Phi(B)^{*} \Phi(C)$ is contained in the weak* closure of $\mathcal{S}+\mathcal{S}^{*}$.

We claim that $\Omega(\Psi(T))=T$ for all $T$ in $\mathcal{T}_{R}$. Indeed, apply Lemma 3.4 to write $T=B^{*} C$ for some $B$ and $C$ in $\mathcal{L}_{n}$, and let $G=\Phi(B)$ and $H=\Phi(C)$. Then we 
have

$$
\begin{aligned}
\Psi(T) & =\Psi\left(B^{*} C\right) \\
& =\Phi(B)^{*} \Phi(C) \\
& =G^{*} H
\end{aligned}
$$

which gives

$$
\begin{aligned}
\Omega(\Phi(T)) & =\Omega\left(G^{*} H\right) \\
& =\left(\Phi^{-1}(G)\right)^{*} \Phi^{-1}(H) \\
& =B^{*} C \\
& =T .
\end{aligned}
$$

Then

which gives

$$
\frac{\|T\|}{\|\Psi(T)\|}=\frac{\|\Omega(\Psi(T))\|}{\|\Psi(T)\|} \leq 1
$$

$$
\|T\| \leq\|\Psi(T)\| \leq\|T\|,
$$

and shows that $\Psi$ maps $\mathcal{T}_{R}$ isometrically onto its range.

We now show that $\Psi$ is weak*-to-weak* continuous. Since the predual of $\mathcal{T}_{R}$ is separable, by an application of the Krein-Smulian theorem it suffices to show that if $T_{n}$ is a sequence in $\mathcal{T}_{R}$ which is weak*-convergent to zero, then $\Psi\left(T_{n}\right)$ is weak* convergent to zero.

Let $\mathcal{A}=\left\{A \oplus \Phi(A): A \in \mathcal{L}_{n}\right\}$, and note that $\mathcal{A}$ is the free semigroup algebra generated by the isometries $L_{1} \oplus S_{1}, \ldots, L_{n} \oplus S_{n}$. Fix $u$ in $\mathcal{H}$. By Theorem 1.6 of [10], there exists a vector $x$ in $\mathcal{F}_{n}$ such that the restriction of $\mathcal{A}$ to $\mathcal{W}=\mathcal{A}[x \oplus u]$ is unitarily equivalent to $\mathcal{L}_{n}$. Letting $P$ denote the projection of $\mathcal{F} \oplus \mathcal{H}$ onto $\mathcal{W}$, and letting $\mathcal{K}$ denote the weak* closure of the restriction of $P\left(\mathcal{A}+\mathcal{A}^{*}\right) P$ to $\mathcal{W}$, it follows that $\mathcal{K}$ is unitarily equivalent to $\mathcal{T}_{R}$. By Lemma 3.4, every element of $\mathcal{K}$ can be written as the restriction to $\mathcal{W}$ of an element of the form

$$
P\left(B^{*} \oplus \Phi(B)^{*}\right)(C \oplus \Phi(C)) P=P\left(B^{*} C \oplus \Psi\left(B^{*} C\right)\right) P .
$$

Hence $\mathcal{K}$ is the restriction to $\mathcal{W}$ of $\left\{T \oplus \psi(T): T \in \mathcal{T}_{R}\right\}$.

If $T_{n}$ is weak* convergent to zero in $\mathcal{T}_{R}$, the unitary equivalence between $\mathcal{T}_{R}$ and $\mathcal{K}$ implies the restriction of the sequence $T_{n} \oplus \Psi\left(T_{n}\right)$ to $\mathcal{W}$ is weak ${ }^{*}$-convergent to zero in $\mathcal{K}$. Hence

$$
\left(\left(T_{n} \oplus \Psi\left(T_{n}\right)\right)(x \oplus u), x \oplus u\right)=\left(T_{n} x, x\right)+\left(\Psi\left(T_{n}\right) u, u\right) \rightarrow 0,
$$

and since $\left(T_{n} x, x\right) \rightarrow 0$, this implies that $\left(\Psi\left(T_{n}\right) u, u\right) \rightarrow 0$. Since $u$ was chosen arbitrarily, we deduce that $\left(\Psi\left(T_{n}\right) u, u\right) \rightarrow 0$ for all $u$ in $\mathcal{H}$. By the polarization identity, we get that $\Psi\left(T_{n}\right)$ is weak operator convergent to zero. By the uniform boundedness principle, the sequence $\Psi\left(T_{n}\right)$ is bounded. It follows that $\Psi\left(T_{n}\right)$ is weak* convergent to zero. We therefore conclude that $\Psi$ is weak* continuous.

It now follows by another application of the Krein-Smulian theorem that $\Psi$ has weak* ${ }^{*}$ closed range, and that $\Psi$ is a weak*-to-weak* homeomorphism onto its range. But it's clear that the range of $\Psi$ is weak* dense in the weak* closure of $\mathcal{S}+\mathcal{S}^{*}$, so $\Psi$ maps $\mathcal{T}_{R}$ weak*-to-weak* homeomorphically onto the weak* closure of $\mathcal{S}+\mathcal{S}^{*}$. From above, $\Psi$ is a completely positive isometry, with completely positive inverse $\Omega$. Hence $\Psi$ is completely isometric. 


\section{WANDERING VECTORS}

Let $\mathcal{S}$ be a weak*-closed subspace of $\mathcal{B}(\mathcal{H})$, and let $x$ and $y$ be vectors in $\mathcal{H}$. Then $[x \otimes y]_{\mathcal{S}}$ denotes the weak-operator-continuous linear functional on $\mathcal{S}$ which is given by the coset of the rank one tensor $x \otimes y$. In other words,

$$
\left(A,[x \otimes y]_{\mathcal{S}}\right)=(A x, y), \quad A \in \mathcal{S} .
$$

Definition 4.1. A weak*-closed subspace $\mathcal{S}$ of $\mathcal{B}(\mathcal{H})$ is said to have property $\mathbb{A}_{1}(1)$ if, for every weak*-continuous linear functional $\pi$ on $\mathcal{S}$ and every $\varepsilon>0$, there are vectors $x$ and $y$ in $\mathcal{H}$ with $\|x\|\|y\|<(1+\epsilon)\|\pi\|$ such that $\pi(A)=(A x, y)$ for all $A$ in $\mathcal{S}$.

It was shown in [12] that $\mathcal{L}_{n}$ has property $\mathbb{A}_{1}(1)$. A result of Bercovici [3] implies that a singly generated type $L$ free semigroup algebra has property $\mathbb{A}_{1}(1)$. In this section, we will use dual algebra techniques to show that every type L free semigroup algebra with $n \geq 2$ generators has property $\mathbb{A}_{1}(1)$. From this result, it will follow easily that every type $\mathrm{L}$ free semigroup algebra has a wandering vector.

For the remainder of this section we fix a type L free semigroup algebra $\mathcal{S}$ with $n \geq 2$ generators acting on a Hilbert space $\mathcal{H}$. The general outline of our approach is as follows. Let $\pi$ be a weak*-continuous linear functional on $\mathcal{S}$. We will show that we can construct convergent sequences $\left(x_{k}\right)$ and $\left(y_{k}\right)$ such that

$$
\lim _{k \rightarrow \infty}\left\|\pi-\left[x_{k} \otimes y_{k}\right]_{\mathcal{S}}\right\|=0 .
$$

This will then give $\pi=[x \otimes y]_{\mathcal{S}}$, where $x=\lim _{k} x_{k}$ and $y=\lim _{k} y_{k}$.

The following idea will allow us to iteratively construct the sequences $\left(x_{k}\right)$ and $\left(y_{k}\right)$. Fix $x_{k}$ and $y_{k}$. Suppose we can find vectors $x^{\prime}$ and $y^{\prime}$ such that

(1) $\left[x^{\prime} \otimes y^{\prime}\right]_{\mathcal{S}}$ approximates the error $\pi-\left[x_{k} \otimes y_{k}\right]_{\mathcal{S}}$ arbitrarily closely,

(2) $\left\|\left[x_{k} \otimes y^{\prime}\right]_{\mathcal{S}}\right\|$ and $\left\|\left[x^{\prime} \otimes y_{k}\right]_{\mathcal{S}}\right\|$ are arbitrarily small,

(3) $\left\|x^{\prime}\right\|$ and $\left\|y^{\prime}\right\|$ are arbitrarily close to $\left\|\pi-\left[x_{k} \otimes y_{k}\right]_{\mathcal{S}}\right\|$.

Set $x_{k+1}=x_{k}+x^{\prime}$ and $y_{k+1}=y_{k}+y^{\prime}$. Then

$\left\|\pi-\left[x_{k+1} \otimes y_{k+1}\right]_{\mathcal{S}}\right\| \leq\left\|\pi-\left[x_{k} \otimes y_{k}\right]_{\mathcal{S}}-\left[x^{\prime} \otimes y^{\prime}\right]_{\mathcal{S}}\right\|+\left\|\left[x^{\prime} \otimes y_{k}\right]_{\mathcal{S}}\right\|+\left\|\left[x_{k} \otimes y^{\prime}\right]_{\mathcal{S}}\right\|$, so $\left[x_{k+1} \otimes y_{k+1}\right]_{\mathcal{S}}$ is an arbitrarily good approximation to $\pi$, and the sequences $\left(x_{k}\right)$ and $\left(y_{k}\right)$ can be made Cauchy. Of course, the main difficulty will be in showing that it is possible to find $x^{\prime}$ and $y^{\prime}$ as above.

Definition 4.2. An operator $X: \mathcal{F}_{n} \rightarrow \mathcal{H}$ is said to intertwine $\mathcal{L}_{n}$ and $\mathcal{S}$ if $X L_{i}=S_{i} X$ for $1 \leq i \leq n$.

Let $\bar{x}=\left(x_{1}, \ldots, x_{m}\right)$ be a wandering vector for $\mathcal{S}^{(m)}$. We know that the restriction of $\mathcal{S}^{(m)}$ to $\mathcal{S}^{(m)}[\bar{x}]$ is unitarily equivalent to $\mathcal{L}_{n}$. Let $X: \mathcal{F}_{n} \rightarrow \mathcal{H}$ denote the map which follows this equivalence with the projection onto the first coordinate. Then $X$ intertwines $\mathcal{L}_{n}$ and $\mathcal{S}$. It was shown in [11] that every vector in $\mathcal{H}$ is in the range of some intertwining operator of this form.

The following result shows that every intertwining operator gives rise to an $L$ Toeplitz operator. This allows us to use the results of section 3 to work with intertwining operators.

Lemma 4.3. Suppose $X: \mathcal{F}_{n} \rightarrow \mathcal{H}$ intertwines $\mathcal{L}_{n}$ and $\mathcal{S}$. Then $X^{*} X$ is an L-Toeplitz operator. 
WANDERING VECTORS AND THE REFLEXIVITY OF FREE SEMIGROUP ALGEBRAS 10

Proof. This follows immediately from the identity

$$
\begin{aligned}
L_{i}^{*} X^{*} X L_{j} & =X^{*} S_{i}^{*} S_{j} X \\
& = \begin{cases}X^{*} X & \text { if } i=j, \\
0 & \text { otherwise. }\end{cases}
\end{aligned}
$$

We require several technical results about $L$-Toeplitz operators.

Lemma 4.4. Let $T$ be an L-Toeplitz operator with Fourier series

$$
T \sim \sum_{w \in \mathbb{F}_{n}^{+}} a_{w} R_{w}+\sum_{w \in \mathbb{F}_{n}^{+} \backslash\{\varnothing\}} \overline{b_{w}} R_{w}^{*} .
$$

Then for any word $u$ in $\mathbb{F}_{n}^{+}$,

$$
\left\|\sum_{w \in \mathbb{F}_{n}^{+} \backslash\{\varnothing\}} \overline{b_{w}} R_{w}^{*} \xi_{u}\right\| \leq\left\|\sum_{w \in \mathbb{F}_{n}^{+} \backslash\{\varnothing\}} b_{w} R_{w} \xi_{u}\right\| .
$$

Proof. We have

$$
\begin{aligned}
\left\|\sum_{w \in \mathbb{F}_{n}^{+} \backslash\{\varnothing\}} \overline{b_{w}} R_{w}^{*} \xi_{u}\right\|^{2} & =\sum_{\substack{w \in \mathbb{F}_{n}^{+} \backslash\{\varnothing\} \\
w=w^{\prime} u}}\left|b_{w}\right|^{2} \\
& \leq \sum_{\substack{w \in \mathbb{F}_{n}^{+} \backslash\{\varnothing\} \\
\left|b_{w}\right|^{2}}} \\
& =\left\|\sum_{w \in \mathbb{F}_{n}^{+} \backslash\{\varnothing\}} b_{w} R_{w} \xi_{u}\right\|^{2} .
\end{aligned}
$$

Lemma 4.5. Let $T$ be an L-Toeplitz operator with Fourier series

$$
T \sim \sum_{w \in \mathbb{F}_{n}^{+}} a_{w} R_{w}+\sum_{w \in \mathbb{F}_{n}^{+} \backslash\{\varnothing\}} \overline{b_{w}} R_{w}^{*} .
$$

Then given $p \geq 1$ and $\epsilon>0$, there is a word $v$ in $\mathbb{F}_{n}^{+}$such that

$$
\left\|R_{v}^{*} T R_{v} \xi_{u}-a_{\varnothing} \xi_{u}\right\|<\epsilon
$$

for any word $u \in \mathbb{F}_{n}^{p}$.

Proof. For $k \geq 1$, let $v_{k}$ be the word $v_{k}=12^{k}$. Then for any word $w$ in $\mathbb{F}_{n}^{+}$,

$$
R_{v_{k}}^{*} R_{w} R_{v_{k}}= \begin{cases}I & \text { if } w=\varnothing, \\ R_{v_{k} w^{\prime}} & \text { if } w=w^{\prime} v_{k} \text { for } w^{\prime} \in \mathbb{F}_{n}^{+}, \\ 0 & \text { otherwise. }\end{cases}
$$

This implies the Fourier series for $R_{v_{k}}^{*} T R_{v_{k}}$ is given by

$$
R_{v_{k}}^{*} T R_{v_{k}} \sim a_{\varnothing} I+\sum_{\substack{w \in \mathbb{F}_{n}^{+} \backslash\{\varnothing\} \\ w=w^{\prime} v_{k}}} a_{w} R_{v_{k} w^{\prime}}+\sum_{\substack{w \in \mathbb{F}_{n}^{+} \backslash\{\varnothing\} \\ w=w^{\prime} v_{k}}} \overline{b_{w}} R_{v_{k} w^{\prime}}^{*} .
$$


WANDERING VECTORS AND THE REFLEXIVITY OF FREE SEMIGROUP ALGEBRAS 11

Hence for $u$ in $\mathbb{F}_{n}^{+}$,

$$
R_{v_{k}}^{*} T R_{v_{k}} \xi_{u} \sim a_{\varnothing} \xi_{u}+\sum_{\begin{array}{c}
w \in \mathbb{F}_{n}^{+} \backslash\{\varnothing\} \\
w=w^{\prime} v_{k}
\end{array}} a_{w} R_{v_{k} w^{\prime}} \xi_{u}+\sum_{\substack{w \in \mathbb{F}_{n}^{+} \backslash\{\varnothing\} \\
w=w^{\prime} v_{k}}} \overline{b_{w}} R_{v_{k} w^{\prime}}^{*} \xi_{u} .
$$

This gives

$$
\begin{aligned}
\left\|R_{v_{k}}^{*} T R_{v_{k}} \xi_{u}-a_{\varnothing} \xi_{u}\right\| & =\left\|\sum_{\substack{w \in \mathbb{F}_{n}^{+} \backslash\{\varnothing\} \\
w=w^{\prime} v_{k}}} a_{w} R_{v_{k} w^{\prime}} \xi_{u}+\sum_{\substack{w \in \mathbb{F}_{n}^{+} \backslash\{\varnothing\} \\
w=w^{\prime} v_{k}}} \overline{b_{w}} R_{v_{k} w^{\prime}}^{*} \xi_{u}\right\| \\
& \leq\left\|\sum_{\substack{w \in \mathbb{F}_{n}^{+} \backslash\{\varnothing\} \\
w=w^{\prime} v_{k}}} a_{w} R_{v_{k} w^{\prime}} \xi_{u}\right\|+\left\|\sum_{\substack{w \in \mathbb{F}_{n}^{+} \backslash\{\varnothing\} \\
w=w^{\prime} v_{k}}} \overline{b_{w}} R_{v_{k} w^{\prime}}^{*} \xi_{u}\right\| \\
& \leq\left\|\sum_{\substack{w \in \mathbb{F}_{n}^{+} \backslash\{\varnothing\} \\
w=w^{\prime} v_{k}}} a_{w} R_{v_{k} w^{\prime}} \xi_{u}\right\|+\left\|\sum_{\substack{w \in \mathbb{F}_{n}^{+} \backslash\{\varnothing\} \\
w=w^{\prime} v_{k}}} b_{w} R_{v_{k} w^{\prime}} \xi_{u}\right\|,
\end{aligned}
$$

where the last inequality follows from Lemma 4.4. Now

$$
\left\|\sum_{\substack{w \in \mathbb{F}_{n}^{+} \backslash\{\varnothing\} \\ w=w^{\prime} v_{k}}} a_{w} R_{v_{k} w^{\prime}} \xi_{u}\right\|^{2}=\sum_{\substack{w \in \mathbb{F}_{n}^{+} \backslash\{\varnothing\} \\ w=w^{\prime} v_{k}}}\left|a_{w}\right|^{2}=\left\|R_{v_{k}}^{*} T \xi_{\varnothing}\right\|^{2},
$$

and similarly,

$$
\left\|\sum_{\substack{w \in \mathbb{F}_{n}^{+} \backslash\{\varnothing\} \\ w=w^{\prime} v_{k}}} b_{w} R_{v_{k} w^{\prime}} \xi_{u}\right\|^{2}=\sum_{\substack{w \in \mathbb{F}_{n}^{+} \backslash\{\varnothing\} \\ w=w^{\prime} v_{k}}}\left|b_{w}\right|^{2}=\left\|R_{v_{k}}^{*} T^{*} \xi_{\varnothing}\right\|^{2},
$$

Hence the result follows from the fact that for all $\xi$ in $\mathcal{F}_{n},\left\|R_{v}^{*} \xi\right\| \rightarrow 0$ as $|v| \rightarrow$ $\infty$.

Recall that $\phi:\left(\mathcal{L}_{n}\right)_{*} \rightarrow \mathcal{S}_{*}$ is the predual of the map $\Phi^{-1}: \mathcal{S} \rightarrow \mathcal{L}_{n}$.

Lemma 4.6. Let $X: \mathcal{F}_{n} \rightarrow \mathcal{H}$ be an intertwining operator, and let $x=X \xi_{\varnothing}$. Then given $p \geq 1$ and $\epsilon>0$, there exists a word $v$ in $\mathbb{F}_{n}^{+}$such that

$$
\left\|\left[S_{u_{1} v} x \otimes S_{u_{2} v} x\right]_{\mathcal{S}}-\right\| x\left\|^{2} \phi\left(\left[\xi_{u_{1}} \otimes \xi_{u_{2}}\right]_{\mathcal{L}_{n}}\right)\right\|<\epsilon
$$

for all words $u_{1}$ and $u_{2}$ in $\mathbb{F}_{n}^{p}$.

Proof. By scaling $X$ if necessary, we can suppose that $\|x\|=1$. Let $T=X^{*} X$. Then $T$ is an $L$-Toeplitz operator by Lemma 4.3. Writing the Fourier series for $T$ as

$$
T \sim \sum_{w \in \mathbb{F}_{n}^{+}} a_{w} R_{w}+\sum_{w \in \mathbb{F}_{n}^{+} \backslash\{0\}} \overline{b_{w}} R_{w}^{*}
$$


it follows that $a_{\varnothing}=\|x\|^{2}=1$. Hence by Lemma 4.5, there exists a word $v$ in $\mathbb{F}_{n}^{+}$ such that $\left\|R_{v}^{*} T R_{v} \xi_{u_{2}}-\xi_{u_{2}}\right\|<\epsilon$ for any word $u_{2}$ in $\mathbb{F}_{n}^{p}$. Then for $A$ in $\mathcal{S}$,

$$
\begin{aligned}
\left(A,\left[S_{u_{1} v} x \otimes S_{u_{2} v} x\right]_{\mathcal{S}}\right) & =\left(A S_{u_{1} v} x, S_{u_{2} v} x\right) \\
& =\left(A S_{u_{1} v} X \xi_{\varnothing}, S_{u_{2} v} X \xi_{\varnothing}\right) \\
& =\left(X \Phi^{-1}(A) L_{u_{1} v} \xi_{\varnothing}, X L_{u_{2} v} \xi_{\varnothing}\right) \\
& =\left(X \Phi^{-1}(A) R_{v} \xi_{u_{1}}, X R_{v} \xi_{u_{2}}\right) \\
& =\left(X R_{v} \Phi^{-1}(A) \xi_{u_{1}}, X R_{v} \xi_{u_{2}}\right) \\
& =\left(\Phi^{-1}(A) \xi_{u_{1}}, R_{v}^{*} T R_{v} \xi_{u_{2}}\right)
\end{aligned}
$$

for all words $u_{1}$ and $u_{2}$ in $\mathbb{F}_{n}^{p}$. This gives

$$
\begin{aligned}
\left|\left(A,\left[S_{u_{1} v} x \otimes S_{u_{2} v} x\right]_{\mathcal{S}}-\left[\xi_{u_{1}} \otimes \xi_{u_{2}}\right]_{\mathcal{L}_{n}}\right)\right| & =\left|\left(\Phi^{-1}(A) \xi_{u_{1}}, R_{v}^{*} T R_{v} \xi_{u_{2}}-\xi_{u_{2}}\right)\right| \\
& \leq\left\|\Phi^{-1}(A) \xi_{u_{1}}\right\|\left\|R_{v}^{*} T R_{v} \xi_{u_{2}}-\xi_{u_{2}}\right\| \\
& <\epsilon\|A\| .
\end{aligned}
$$

Therefore,

$$
\left\|\left[S_{u_{1} v} x \otimes S_{u_{2} v} x\right]_{\mathcal{S}}-\phi\left(\left[\xi_{u_{1}} \otimes \xi_{u_{2}}\right]_{\mathcal{L}_{n}}\right)\right\|<\epsilon .
$$

Lemma 4.7. Let $M \geq 2$ be minimal such that $\mathcal{S}^{(M)}$ has a wandering vector $\bar{w}=$ $\left(w_{1}, \ldots, w_{M}\right)$. Then given $\epsilon \in(0,1)$ there exists a unit vector $\bar{x}=\left(x_{1}, \ldots, x_{M}\right)$ in $\mathcal{S}^{(M)}[\bar{w}]$ such that $x_{1}=X \xi_{\varnothing}$ for some intertwining operator $X: \mathcal{F}_{n} \rightarrow \mathcal{H}$, and $\left\|x_{1}\right\|>1-\epsilon$.

Proof. Let $P$ denote the projection map from $\mathcal{S}^{(M)}[\bar{w}]$ to $\mathcal{H}^{(M-1)}$ which takes $\bar{x}=\left(x_{1}, \ldots, x_{M}\right)$ to $\left(x_{2}, \ldots, x_{M}\right)$. Then $P$ intertwines the restriction of $\mathcal{S}^{(M)}$ to $\mathcal{S}^{(M)}[\bar{w}]$ and $\mathcal{S}^{(M-1)}$. The restriction of $\mathcal{S}^{(M)}$ to $\mathcal{S}^{(M)}[\bar{w}]$ is unitarily equivalent to $\mathcal{L}_{n}$. Let $U$ be a unitary implementing this equivalence. Then setting $Y=P U, Y$ intertwines $\mathcal{L}_{n}$ and $S^{(M-1)}$. Suppose that for all $\bar{x}$ in $\mathcal{S}^{(M)}[\bar{w}],\left\|x_{1}\right\| \leq(1-\epsilon)\|\bar{x}\|$. Then

which gives

$$
\|\bar{x}\|^{2}=\sum_{i=1}^{M}\left\|x_{i}\right\|^{2} \leq(1-\epsilon)^{2}\|\bar{x}\|^{2}+\sum_{i=2}^{M}\left\|x_{i}\right\|^{2},
$$

$$
\sum_{i=2}^{m M}\left\|x_{i}\right\|^{2} \geq\left(1-(1-\epsilon)^{2}\right)\|\bar{x}\|^{2},
$$

implying that $P$ is bounded below, and hence that $Y$ is bounded below. By [11, Theorem 2.8], this implies that the range of $Y$ is a wandering subspace for $\mathcal{S}^{(M-1)}$, contradicting the minimality of $M$. Hence there must be some unit vector $\bar{x}$ in $\mathcal{S}^{(M)}[\bar{w}]$ such that $\left\|x_{1}\right\|>1-\epsilon$.

Let $Q$ denote the projection map from $\mathcal{S}^{(M)}[\bar{w}]$ to $\mathcal{H}$ which takes $\bar{y}=\left(y_{1}, \ldots, y_{M}\right)$ to $\bar{y}_{1}$, and let $Z=Q U$. Note that $x_{1}$ is contained in the range of $Z$. For every $R$ in $\mathcal{R}_{n}$, the operator $Z R$ intertwines $\mathcal{L}_{n}$ and $\mathcal{S}$. Moreover, since the set of vectors $\left\{R \xi_{\varnothing}: R \in \mathcal{R}_{n}\right\}$ is dense in $\mathcal{F}_{n}$, the set $\left\{Z R \xi_{\varnothing}: R \in \mathcal{R}_{n}\right\}$ is dense in the closure of the range of $Z$. It follows that we can choose the vector $\bar{x}$ as above such that $x_{1}=X \xi_{\varnothing}$ for some intertwining operator $X: \mathcal{F}_{n} \rightarrow \mathcal{H}$.

Let $M \geq 1$ be minimal such that the ampliation $\mathcal{S}^{(M)}$ has a wandering vector $\bar{x}$. Such $M$ exists by Theorem 2.1. Then $\mathcal{H}^{(M)}$ contains an infinite family of pairwise orthogonal subspaces $\mathcal{W}_{k}$, for $k \geq 1$, which are wandering for $\mathcal{S}^{(M)}$. For example, 
we can take $\mathcal{W}_{k}=\mathcal{S}^{(m)}\left[S_{v_{k}}^{(m)} \bar{x}\right]$, where $v_{k}=12^{k}$. For $k \geq 1$, let $\mathcal{M}_{k}$ denote the linear manifold in $\mathcal{H}$ given by

$$
\mathcal{M}_{k}=\left\{z \in \mathcal{H}: z=\bar{z}_{1} \text { for some } \bar{z}=\left(\bar{z}_{1}, \ldots, \bar{z}_{M}\right) \text { in } \mathcal{W}_{k}\right\}
$$

Let $\mathcal{W}$ denote the algebraic span of the $\mathcal{W}_{k}$, and let $\mathcal{M}$ denote the algebraic span of the $\mathcal{M}_{k}$.

Lemma 4.8. Given $h_{1}, \ldots, h_{q}$ in $\mathcal{M}$ and $\epsilon>0$, there exists a unit vector $y$ in $\mathcal{M}$ such that $y=Y \xi_{\varnothing}$ for some intertwining operator $Y: \mathcal{F}_{n} \rightarrow \mathcal{H}$, and such that $\left\|\left[S_{u} y \otimes h_{j}\right]_{\mathcal{S}}\right\|<\epsilon$ and $\left\|\left[h_{j} \otimes S_{u} y\right]_{\mathcal{S}}\right\|<\epsilon$ for any word $u \in \mathbb{F}_{n}^{+}$and $1 \leq j \leq q$.

Proof. For each $j$, there exists $\bar{h}^{(j)}=\left(h_{1}^{(j)}, \ldots, h_{M}^{(j)}\right)$ in $\mathcal{W}$ such that $h_{j}=h_{1}^{(j)}$. Choose $\epsilon_{0} \in(0,1)$ such that $\epsilon_{0} /\left(1-\epsilon_{0}\right)<\epsilon$ and $\epsilon_{0} /\left\|h^{(j)}\right\|<1$ for $1 \leq j \leq q$, and choose $r$ sufficiently large that $\bar{h}^{(j)}$ is orthogonal to $\mathcal{W}_{r}$ for $1 \leq j \leq q$.

By Lemma 4.7, there exists a unit vector $\bar{x}=\left(x_{1}, \ldots, x_{M}\right)$ in $\mathcal{M}_{r}$ such that $x_{1}=X \xi_{\varnothing}$ for some intertwining operator $X: \mathcal{F}_{n} \rightarrow \mathcal{H}$, and such that

$$
\left\|x_{1}\right\|>\max \left\{1-\epsilon_{0},\left(1-\frac{\epsilon_{0}^{2}}{\left\|\bar{h}^{(j)}\right\|^{2}}\right)^{1 / 2}: 1 \leq j \leq q\right\} .
$$

This gives $1 /\left\|x_{1}\right\|<1 /\left(1-\epsilon_{0}\right)$ and

$$
\sum_{i=2}^{M}\left\|x_{i}\right\|^{2}=1-\left\|x_{1}\right\|^{2}<\frac{\epsilon_{0}^{2}}{\left\|\bar{h}^{(j)}\right\|^{2}}, \quad 1 \leq j \leq q .
$$

For any word $u$ in $\mathbb{F}_{n}^{+}$,

$$
\begin{aligned}
\left\|\left[S_{u} x_{1} \otimes h_{1}^{(j)}\right]_{\mathcal{S}}\right\| & \leq\left\|\sum_{i=1}^{M}\left[S_{u} x_{i} \otimes h_{i}^{(j)}\right]_{\mathcal{S}}\right\|+\left\|\sum_{i=2}^{M}\left[S_{u} x_{i} \otimes h_{i}^{(j)}\right]_{\mathcal{S}}\right\| \\
& =\left\|\left[S_{u}^{(M)} \bar{x} \otimes \bar{h}^{(j)}\right]_{\mathcal{S}}\right\|+\left\|\sum_{i=2}^{M}\left[S_{u} x_{i} \otimes h_{i}^{(j)}\right]_{\mathcal{S}}\right\| \\
& =\left\|\sum_{i=2}^{M}\left[S_{u} x_{i} \otimes h_{i}^{(j)}\right]_{\mathcal{S}}\right\| \\
& \leq\left(\sum_{i=2}^{M}\left\|S_{u} x_{i}\right\|^{2}\right)^{1 / 2}\left(\sum_{i=2}^{M}\left\|h_{i}^{(j)}\right\|^{2}\right)^{1 / 2} \\
& =\left(\sum_{i=2}^{M}\left\|x_{i}\right\|^{2}\right)^{1 / 2}\left(\sum_{i=2}^{M}\left\|h_{i}^{(j)}\right\|^{2}\right)^{1 / 2} \\
& <\frac{\epsilon_{0}}{\left\|\bar{h}^{(j)}\right\|}\left(\sum_{i=2}^{M}\left\|h_{i}^{(j)}\right\|^{2}\right)^{1 / 2} \\
& \leq \epsilon_{0},
\end{aligned}
$$

where we have used the fact that $\bar{x}$ and $\bar{h}^{(j)}$ belong to orthogonal $\mathcal{S}^{(M)}$-invariant subspaces, which implies that $\left\|\left[S_{u}^{(M)} \bar{x} \otimes \bar{h}^{(j)}\right]_{\mathcal{S}}\right\|=0$. Multiplying this inequality by $1 /\left\|x_{1}\right\|=1 /\left\|S_{u} x_{1}\right\|$ then gives

$$
\left\|\left[S_{u}\left(x_{1} /\left\|x_{1}\right\|\right) \otimes h_{j}\right]\right\|<\epsilon_{0} /\left(1-\epsilon_{0}\right)
$$


WANDERING VECTORS AND THE REFLEXIVITY OF FREE SEMIGROUP ALGEBRAS 14

for $1 \leq j \leq q$. In the same way we get

$$
\left.\| h_{j} \otimes S_{u}\left(x_{1} /\left\|x_{1}\right\|\right)\right] \|<\epsilon_{0} /\left(1-\epsilon_{0}\right)
$$

for $1 \leq j \leq q$. Hence we can take $y=x_{1} /\left\|x_{1}\right\|$ and $Y=X /\left\|x_{1}\right\|$.

Lemma 4.9. Given $h_{1}, \ldots, h_{q}$ in $\mathcal{M}, p \geq 1$, and $\epsilon>0$, there exists a unit vector $z$ in $\mathcal{M}$ such that

$$
\left\|\left[S_{u_{1}} z \otimes S_{u_{2}} z\right]_{\mathcal{S}}-\phi\left(\left[\xi_{u_{1}} \otimes \xi_{u_{2}}\right]_{\mathcal{L}_{n}}\right)\right\|<\epsilon
$$

for all $u_{1}$ and $u_{2}$ in $\mathbb{F}_{n}^{p}$, and such that $\left\|\left[S_{w} z \otimes h_{j}\right]_{\mathcal{S}}\right\|<\epsilon$ and $\left\|\left[h_{j} \otimes S_{w} z\right]_{\mathcal{S}}\right\|<\epsilon$ for all $w \in \mathbb{F}_{n}^{+}$and $1 \leq j \leq q$.

Proof. By Lemma 4.8, there exists a unit vector $y$ in $\mathcal{M}$ such that $y=Y \xi_{\varnothing}$ for some intertwining operator $Y: \mathcal{F}_{n} \rightarrow \mathcal{H}$, and such that for any word $w$ in $\mathbb{F}_{n}^{+}$, $\left\|\left[S_{w} y \otimes h_{j}\right]_{\mathcal{S}}\right\|<\epsilon$ and $\left\|\left[h_{j} \otimes S_{w} y\right]_{\mathcal{S}}\right\|<\epsilon$ for $1 \leq j \leq q$. By Lemma 4.6, there exists a word $v$ in $\mathbb{F}_{n}^{+}$such that $\left\|\left[S_{u_{1}} S_{v} y \otimes S_{u_{2}} S_{v} y\right]_{\mathcal{S}}-\phi\left(\left[\xi_{u_{1}} \otimes \xi_{u_{2}}\right]_{\mathcal{L}_{n}}\right)\right\|<\epsilon$ for any words $u_{1}$ and $u_{2}$ in $\mathbb{F}_{n}^{p}$. Then $\left\|\left[S_{w} S_{v} y \otimes h_{j}\right]_{\mathcal{S}}\right\|=\left\|\left[S_{w v} y \otimes h_{j}\right]_{\mathcal{S}}\right\|<\epsilon$ and $\left\|\left[h_{j} \otimes S_{w} S_{v} y\right]_{\mathcal{S}}\right\|=\left\|\left[h_{j} \otimes S_{w v} y\right]_{\mathcal{S}}\right\|<\epsilon$, so we can take $z=S_{v} y$.

Lemma 4.10. Given a weak ${ }^{*}$-continuous linear functional $\pi$ on $\mathcal{S}, h_{1}, \ldots, h_{q}$ in $\mathcal{M}$, and $\epsilon>0$, there are vectors $x$ and $y$ in $\mathcal{M}$ such that

(1) $\left\|\pi-[x \otimes y]_{\mathcal{S}}\right\|<\epsilon$,

(2) $\|x\|<(1+\epsilon)\|\pi\|^{1 / 2}$ and $\|y\|<(1+\epsilon)\|\pi\|^{1 / 2}$,

(3) $\left\|\left[x \otimes h_{j}\right]_{\mathcal{S}}\right\|<\epsilon$ and $\left\|\left[h_{j} \otimes y\right]_{\mathcal{S}}\right\|<\epsilon$ for $1 \leq j \leq q$.

Proof. By scaling $\pi$ and $\epsilon$ if necessary, we can assume that $\|\pi\|=1$. Choose $\epsilon_{0}>0$ such that $2 \epsilon_{0}+3 \epsilon_{0}^{2}<\epsilon / 2$ and $4 \epsilon_{0}+4 \epsilon_{0}^{2}<\epsilon+\epsilon^{2} / 2$. Since $\mathcal{L}_{n}$ has property $\mathbb{A}_{1}(1)$, there are vectors $\xi$ and $\eta$ in $\mathcal{F}_{n}$ such that $[\xi \otimes \eta]_{\mathcal{L}_{n}}=\phi^{-1}(\pi)$, with $\|\xi\|<1+\epsilon_{0}$ and $\|\eta\|<1+\epsilon_{0}$.

Since $\xi_{\varnothing}$ is cyclic for $\mathcal{L}_{n}$, there is $p \geq 1$ and $C$ and $D$ in the span of $\left\{L_{u}: u \in \mathbb{F}_{n}^{p}\right\}$ such that $\left\|C \xi_{\varnothing}-\xi\right\|<\epsilon_{0}$ and $\left\|D \xi_{\varnothing}-\eta\right\|<\epsilon_{0}$. Then

$$
\left\|C \xi_{\varnothing}\right\| \leq\left\|C \xi_{\varnothing}-\xi\right\|+\|\xi\|<1+2 \epsilon_{0}
$$

so $\left\|C \xi_{\varnothing}\right\|^{2}<1+\epsilon+\epsilon^{2} / 2$, and similarly, $\left\|D \xi_{\varnothing}\right\|^{2}<1+\epsilon+\epsilon^{2} / 2$. Also,

$$
\begin{aligned}
\left\|\pi-\left[C \xi_{\varnothing} \otimes D \xi_{\varnothing}\right]_{\mathcal{L}_{n}}\right\| \leq & \left\|[\xi \otimes \eta]_{\mathcal{L}_{n}}-\left[C \xi_{\varnothing} \otimes D \xi_{\varnothing}\right]_{\mathcal{L}_{n}}\right\| \\
\leq & \left\|\left[\left(\xi-C \xi_{\varnothing}\right) \otimes \eta\right]_{\mathcal{L}_{n}}\right\|+\left\|\left[\left(C \xi_{\varnothing}-\xi\right) \otimes\left(\eta-D \xi_{\varnothing}\right)\right]_{\mathcal{L}_{n}}\right\|+ \\
& \left\|\left[\xi \otimes\left(\eta-D \xi_{\varnothing}\right)\right]_{\mathcal{L}_{n}}\right\| \\
\leq & \left\|\xi-C \xi_{\varnothing}\right\|\|\eta\|+\left\|\xi-C \xi_{\varnothing}\right\|\left\|\eta-D \xi_{\varnothing}\right\|+ \\
& \|\xi\|\left\|\eta-D \xi_{\varnothing}\right\| \\
< & 2 \epsilon_{0}+3 \epsilon_{0}^{2} \\
< & \epsilon / 2 .
\end{aligned}
$$

Set $A=\Phi(C)$ and $B=\Phi(D)$. If we expand $C$ and $D$ as

$$
C=\sum_{u \in \mathbb{F}_{n}^{p}} c_{u} L_{u} \quad \text { and } \quad D=\sum_{u \in \mathbb{F}_{n}^{p}} d_{u} L_{u},
$$

then

$$
A=\sum_{u \in \mathbb{F}_{n}^{p}} c_{u} S_{u} \quad \text { and } \quad B=\sum_{u \in \mathbb{F}_{n}^{p}} d_{u} S_{u}
$$


Choose $\epsilon_{1}>0$ such that

$$
\begin{gathered}
\epsilon_{1} \sum_{u \in \mathbb{F}_{n}^{p}}\left|c_{u}\right|<\epsilon, \quad \epsilon_{1} \sum_{u \in \mathbb{F}_{n}^{p}}\left|\overline{d_{u}}\right|<\epsilon, \quad \epsilon_{1} \sum_{u \in \mathbb{F}_{n}^{p}} \sum_{v \in \mathbb{F}_{n}^{p}}\left|c_{u} \overline{d_{v}}\right|<\epsilon / 2, \\
\epsilon_{1} \sum_{u \in \mathbb{F}_{n}^{N}} \sum_{v \in \mathbb{F}_{n}^{N}}\left|c_{u} \overline{c_{v}}\right|<\epsilon+\epsilon^{2} / 2, \quad \epsilon_{1} \sum_{u \in \mathbb{F}_{n}^{N}} \sum_{v \in \mathbb{F}_{n}^{N}}\left|d_{u} \overline{d_{v}}\right|<\epsilon+\epsilon^{2} / 2 .
\end{gathered}
$$

By Lemma 4.9, there exists a unit vector $z$ in $\mathcal{M}$ such that

$$
\left\|\left[S_{u} z \otimes S_{v} z\right]_{\mathcal{S}}-\phi\left(\left[\xi_{u} \otimes \xi_{v}\right]_{\mathcal{L}_{n}}\right)\right\|<\epsilon_{1}
$$

for any words $u$ and $v$ in $\mathbb{F}_{n}^{p}$, and such that $\left\|\left[S_{u} z \otimes h_{j}\right]_{\mathcal{S}}\right\|<\epsilon_{1}$ and $\left\|\left[h_{j} \otimes S_{u} z\right]_{\mathcal{S}}\right\|<\epsilon_{1}$ for any word $u$ in $\mathbb{F}_{n}^{+}$and $1 \leq j \leq q$. Then

$$
\begin{aligned}
\|[A z \otimes B z]_{\mathcal{S}}- & \phi\left(\left[C \xi_{\varnothing} \otimes D \xi_{\varnothing}\right]_{\mathcal{L}_{n}}\right) \| \\
& =\left\|\sum_{u \in \mathbb{F}_{n}^{p}} \sum_{v \in \mathbb{F}_{n}^{p}} c_{u} \overline{d_{v}}\left(\left[S_{u} z \otimes S_{v} z\right]_{\mathcal{S}}-\phi\left(\left[S_{u} \xi_{\varnothing} \otimes S_{v} \xi_{\varnothing}\right] \mathcal{L}_{n}\right)\right)\right\| \\
& \left.\left.\leq \sum_{u \in \mathbb{F}_{n}^{p}} \sum_{v \in \mathbb{F}_{n}^{p}}\left|c_{u} \overline{d_{v}}\right| \|\left[S_{u} z \otimes S_{v} z\right]_{\mathcal{S}}-\phi\left(\left[S_{u} \xi_{\varnothing} \otimes S_{v} \xi_{\varnothing}\right]\right]_{\mathcal{L}_{n}}\right)\right) \| \\
& <\epsilon_{1} \sum_{u \in \mathbb{F}_{n}^{p}} \sum_{v \in \mathbb{F}_{n}^{p}}\left|c_{u} \overline{d_{v}}\right| \\
& <\epsilon / 2 .
\end{aligned}
$$

Hence from above,

$\left\|\pi-[A z \otimes B z]_{\mathcal{S}}\right\| \leq \| \pi-\left[\phi\left(\left[C \xi_{\varnothing} \otimes D \xi_{\varnothing}\right]_{\mathcal{L}_{n}}\right)\|+\| \phi\left(\left[C \xi_{\varnothing} \otimes D \xi_{\varnothing}\right]_{\mathcal{L}_{n}}\right)-[A z \otimes B z]_{\mathcal{S}} \|<\epsilon\right.$.

By a similar estimation,

$$
\left\|[A z \otimes A z]_{\mathcal{S}}-\phi\left(\left[C \xi_{\varnothing} \otimes C \xi_{\varnothing}\right]_{\mathcal{L}_{n}}\right)\right\|<\epsilon_{1} \sum_{u \in \mathbb{F}_{n}^{p}} \sum_{v \in \mathbb{F}_{n}^{p}}\left|c_{u} \overline{c_{v}}\right|<\epsilon+\epsilon^{2} / 2 .
$$

Evaluation of these functionals at the identity then implies

$$
\epsilon+\epsilon^{2} / 2>\|A z\|^{2}-\left\|C \xi_{\varnothing}\right\|^{2} \geq\|A z\|^{2}-\left(1+\epsilon+\epsilon^{2} / 2\right),
$$

and hence that $\|A z\|<1+\epsilon$. In the same way we get $\|B z\|<1+\epsilon$.

Finally,

$$
\begin{aligned}
\left\|\left[A z \otimes h_{j}\right]_{\mathcal{S}}\right\| & =\left\|\sum_{u \in \mathbb{F}_{n}^{p}} c_{u}\left[S_{u} z \otimes h_{j}\right]_{\mathcal{S}}\right\| \\
& \leq \sum_{u \in \mathbb{F}_{n}^{p}}\left|c_{u}\right|\left\|\left[S_{u} z \otimes h_{j}\right]_{\mathcal{S}}\right\| \\
& <\epsilon_{1} \sum_{u \in \mathbb{F}_{n}^{p}}\left|c_{u}\right| \\
& <\epsilon,
\end{aligned}
$$

and in the same way we get

$$
\left\|\left[h_{j} \otimes B z\right]_{\mathcal{S}}\right\|<\epsilon_{1} \sum_{u \in \mathbb{F}_{n}^{p}}\left|\bar{d}_{u}\right|<\epsilon .
$$

Hence we can take $x=A z$ and $y=B z$. 
Theorem 4.11. Given a weak*-continuous linear functional $\pi$ on $\mathcal{S}$ and $\epsilon>0$, there are vectors $x$ and $y$ in $\mathcal{H}$ such that $\pi=[x \otimes y]_{\mathcal{S}},\|x\|<(1+\epsilon)\|\pi\|^{1 / 2}$, and $\|y\|<(1+\epsilon)\|\pi\|^{1 / 2}$. In other words, $\mathcal{S}$ has property $\mathbb{A}_{1}(1)$.

Proof. By scaling $\pi$ if necessary, we can assume that $\|\pi\|=1$. Choose $\alpha>0$ such that $(1+\alpha) /(1-\alpha)<1+\epsilon$. Note that $\alpha^{k} \rightarrow 0$ as $k \rightarrow \infty$. We claim that for $k \geq 1$, we can find $x_{k}$ and $y_{k}$ in $\mathcal{M}$ such that

(1) $\left\|\pi-\left[x_{k} \otimes y_{k}\right]_{\mathcal{S}}\right\|<\alpha^{2 k}$

(2) $\left\|x_{k}\right\|<(1+\alpha)\left(1+\alpha+\ldots+\alpha^{k-1}\right)$ and $\left\|y_{k}\right\|<(1+\alpha)\left(1+\alpha+\ldots+\alpha^{k-1}\right)$,

(3) $\left\|x_{k}-x_{k-1}\right\|<(1+\alpha) \alpha^{k-1}$ and $\left\|y_{k}-y_{k-1}\right\|<(1+\alpha) \alpha^{k-1}$ for $k \geq 2$.

Setting $x_{0}=0$ and $y_{0}=0$, Lemma 4.10 easily implies this is true for $k=1$. Proceeding by induction, suppose that we have found $x_{k}$ and $y_{k}$ satisfying these conditions. Choose $\epsilon_{0}>0$ such that $\epsilon_{0}<\alpha$ and $\epsilon_{0}<\alpha^{2(k+1)} / 3$. By Lemma 4.10, there are $x^{\prime}$ and $y^{\prime}$ in $\mathcal{M}$ such that

(1) $\left\|\pi-\left[x_{k} \otimes y_{k}\right]_{\mathcal{S}}-\left[x^{\prime} \otimes y^{\prime}\right]_{\mathcal{S}}\right\|<\epsilon_{0}$,

(2) $\left\|x^{\prime}\right\|<\left(1+\epsilon_{0}\right)\left\|\pi-\left[x_{k} \otimes y_{k}\right]_{\mathcal{S}}\right\|^{1 / 2}$ and

$\left\|y^{\prime}\right\|<\left(1+\epsilon_{0}\right)\left\|\pi-\left[x_{k} \otimes y_{k}\right]_{\mathcal{S}}\right\|^{1 / 2}$,

(3) $\left\|\left[x^{\prime} \otimes y_{k}\right]_{\mathcal{S}}\right\|<\epsilon_{0}$ and $\left\|\left[x_{k} \otimes y^{\prime}\right]_{\mathcal{S}}\right\|<\epsilon_{0}$.

Set $x_{k+1}=x_{k}+x^{\prime}$, and $y_{k+1}=y_{k}+y^{\prime}$. Then

$$
\begin{aligned}
\left\|\pi-\left[x_{k+1} \otimes y_{k+1}\right]_{\mathcal{S}}\right\| & =\left\|\pi-\left[\left(x_{k}+x^{\prime}\right) \otimes\left(y_{k}+y^{\prime}\right)\right]_{\mathcal{S}}\right\| \\
\leq & \left\|\pi-\left[x_{k} \otimes y_{k}\right]_{\mathcal{S}}-\left[x^{\prime} \otimes y^{\prime}\right]_{\mathcal{S}}\right\|+\left\|\left[x_{k} \otimes y^{\prime}\right]_{\mathcal{S}}\right\|+ \\
& \left\|\left[x^{\prime} \otimes y_{k}\right]_{\mathcal{S}}\right\| \\
< & 3 \epsilon_{0} \\
< & \alpha^{2(k+1)} .
\end{aligned}
$$

Also,

which gives

$$
\left\|x^{\prime}\right\|<\left(1+\epsilon_{0}\right)\left\|\pi-\left[x_{k} \otimes y_{k}\right]_{\mathcal{S}}\right\|^{1 / 2}<(1+\alpha) \alpha^{k},
$$

$$
\left\|x_{k+1}\right\|=\left\|x_{k}+x^{\prime}\right\| \leq\left\|x_{k}\right\|+\left\|x^{\prime}\right\|<(1+\alpha)\left(1+\alpha+\ldots+\alpha^{k}\right)
$$

and

$$
\left\|x_{k+1}-x_{k}\right\|=\left\|x^{\prime}\right\|<(1+\alpha) \alpha^{k} .
$$

Symmetrically, $\left\|y_{k+1}\right\|<(1+\alpha)\left(1+\alpha+\ldots+\alpha^{k}\right)$ and $\left\|y_{k+1}-y_{k}\right\|<(1+\alpha) \alpha^{k}$, which establishes the claim.

Now for $l>k$,

$$
\begin{aligned}
\left\|x_{l}-x_{k}\right\| & \leq\left\|x_{l}-x_{l-1}\right\|+\ldots+\left\|x_{k+1}-x_{k}\right\| \\
& <(1+\alpha)\left(\alpha^{l-1}+\ldots+\alpha^{k}\right) \\
& \leq \alpha^{l-1}(1+\alpha) /(1-\alpha)
\end{aligned}
$$

so the sequence $\left(x_{k}\right)$ is Cauchy. Let $x=\lim _{k} x_{k}$. Then

$$
\|x\|=\lim _{k}\left\|x_{k}\right\| \leq \lim _{k}(1+\alpha)\left(1+\alpha+\ldots+\alpha^{k-1}\right)=(1+\alpha) /(1-\alpha)<1+\epsilon .
$$

Similarly, the sequence $\left\{y_{k}\right\}$ is Cauchy. Letting $y=\lim _{k} y_{k}$ be its limit, $\|y\|<1+\epsilon$. Finally, we have

$$
\left\|\pi-[x \otimes y]_{\mathcal{S}}\right\|=\lim \left\|\pi-\left[x_{k} \otimes y_{k}\right]_{\mathcal{S}}\right\| \leq \lim \alpha^{2 k}=0,
$$

so $\pi=[x \otimes y]_{\mathcal{S}}$. 
Theorem 4.12. Every type $L$ free semigroup algebra has a wandering vector.

Proof. Let $\mathcal{S}$ be a type L free semigroup algebra, and let $\mathcal{S}_{0}$ denote the weakoperator-closed ideal generated by $S_{1}, \ldots, S_{n}$. Since $\mathcal{S}$ is type L, $\mathcal{S}_{0}$ is proper, and in particular doesn't contain the identity. Let $\pi_{0}$ denote the weak-operator continuous linear functional which annihilates $\mathcal{S}_{0}$ and satisfies $\pi(I)=1$.

Since $\mathcal{S}$ has property $\mathbb{A}_{1}(1)$, there are vectors $x$ and $y$ in $\mathcal{H}$ such that $\pi_{0}(A)=$ $(A x, y)$ for all $A$ in $\mathcal{S}$. This implies $\left(S_{w} x, y\right)=0$ for all $w \in \mathbb{F}_{n}^{+} \backslash\{\varnothing\}$, so $y$ is orthogonal to the subspace $\mathcal{S}_{0}[x]$. However, $(x, y)=\pi(I)=1$, so $y$ is not orthogonal to the subspace $\mathcal{S}[x]$. Hence $\mathcal{S}[x] \ominus \mathcal{S}_{0}[x]$ is nonempty.

Let $z$ be a unit vector in $\mathcal{S}[x] \ominus \mathcal{S}_{0}[x]$. Then the subspace $\mathcal{S}_{0}[z]$ is contained in the subspace $\mathcal{S}_{0}[x]$, and in particular, is orthogonal to $z$. Hence $\left(S_{w} z, z\right)=0$ for all $w \in \mathbb{F}_{n}^{+} \backslash\{\varnothing\}$. Let $u$ and $v$ be distinct words in $\mathbb{F}_{n}^{+}$such that $|u| \leq|v|$. Then $S_{u}^{*} S_{v}$ is in $\mathcal{S}_{0}$, so $\left(S_{u} z, S_{v} z\right)=\left(z, S_{u}^{*} S_{v} z\right)=0$. By symmetry, it follows that $\left(S_{u} z, S_{v} z\right)=0$ for every pair of distinct words $u$ and $v$ in $\mathbb{F}_{n}^{+}$. Thus $z$ is a wandering vector for $\mathcal{S}$.

Corollary 4.13. A free semigroup algebra is either a von Neumann algebra, or it contains a wandering vector.

Proof. Let $\mathcal{S}$ be a free semigroup algebra. By the general structure theorem for free semigroup algebras [10], $\mathcal{S}$ is either a von Neumann algebra, or it has a type L part. In the latter case, by Theorem 4.12, $\mathcal{S}$ has a wandering vector.

By Theorem 4.1 of [11], every free semigroup algebra which has a wandering vector is reflexive. Thus we have established the following result.

Corollary 4.14. Every free semigroup algebra is reflexive.

Theorem 4.2 of [11] shows that every type L free semigroup algebra which has a wandering vector is hyper-reflexive with hyper-reflexivity constant at most 55 . This gives the following result, which we will refine in section 5 .

Corollary 4.15. Every type L free semigroup algebra is hyper-reflexive with hyperreflexivity constant at most 55 .

\section{Hyper-Reflexivity and the Factorization of Linear Functionals}

In this section we will show that the predual of every type L free semigroup algebra with $n \geq 2$ generators satisfies a very strong factorization property. By a result of Bercovici [4], we will obtain as a consequence that every such algebra is hyper-reflexive with hyper-reflexivity constant at most 3 .

Definition 5.1. A weak*-closed subspace $\mathcal{S}$ of $\mathcal{B}(\mathcal{H})$ is said to have property $\mathcal{X}_{0,1}$ if given a weak*-continuous linear functional $\pi$ on $\mathcal{S}$ with $\|\pi\| \leq 1, h_{1}, \ldots, h_{q}$ in $\mathcal{H}$, and $\epsilon>0$, there are vectors $x$ and $y$ in $\mathcal{H}$ such that

(1) $\left\|\pi-[x \otimes y]_{\mathcal{S}}\right\|<\epsilon$,

(2) $\|x\| \leq 1$ and $\|y\| \leq 1$,

(3) $\left\|\left[x \otimes h_{j}\right]_{\mathcal{S}}\right\|<\epsilon$ and $\left\|\left[h_{j} \otimes y\right]_{\mathcal{S}}\right\|<\epsilon$ for $1 \leq j \leq q$.

Bercovici [4] showed that any weak*-closed algebra whose commutant contains two isometries with pairwise orthogonal ranges has property $\mathcal{X}_{0,1}$, and showed that any weak ${ }^{*}$-closed algebra with propety $\mathcal{X}_{0,1}$ is hyper-reflexive with hyper-reflexivity 
constant at most 3 . For $n \geq 2$, this includes $\mathcal{L}_{n}$. We will show that every type $\mathrm{L}$ free semigroup algebra with $n \geq 2$ generators has property $\mathcal{X}_{0,1}$.

We require the following result which is implied by Lemma 1.2 in [14].

Lemma 5.2. Given an isometry $V$ in $\mathcal{R}_{n}$, vectors $\nu_{1}, \ldots, \nu_{q}$ in $\mathcal{F}_{n}$, and $\epsilon>0$, there exists $m$ such that $\left\|\left(V^{*}\right)^{m} \eta_{j}\right\|<\epsilon$ for $1 \leq j \leq q$.

For the remainder of this section we fix a type L free semigroup algebra $\mathcal{S}$ with $n \geq 2$ generators acting on a Hilbert space $\mathcal{H}$, and we let $\mathcal{Z}$ denote the weak* closure of $\mathcal{S}+\mathcal{S}^{*}$. Let $\Phi$ denote the canonical map from $\mathcal{L}_{n}$ to $\mathcal{S}$. By Theorem 3.6, we can extend $\Phi$ to a map from the set $\mathcal{T}_{R}$ of $R$-Toeplitz operators to $\mathcal{Z}$, and this extension is a complete isometry and a weak*-to-weak* homeomorphism.

For $x$ and $y$ in $\mathcal{H}$, we will need to take care to distinguish between the weakoperator-continuous vector functional $[x \otimes y]_{\mathcal{S}}$ defined on $\mathcal{S}$, and the weak-operatorcontinuous vector functional $[x \otimes y]_{\mathcal{Z}}$ defined on $\mathcal{Z}$.

The following lemma is a variation of an argument of Bercovici [4]. It was kindly provided by Ken Davidson.

Lemma 5.3. Given isometries $U$ and $V$ in $\mathcal{R}_{n}$ with orthogonal ranges, vectors $\xi$ and $\nu$ in $\mathcal{F}_{n}$ with $\nu$ in the kernel of $U^{*}$, and $\epsilon>0$, define

$$
\eta_{k}=\frac{1}{\sqrt{k}} \sum_{i=1}^{k} U^{i} V \xi
$$

Then $\lim _{k}\left\|\left[\nu \otimes \eta_{k}\right]_{\mathcal{T}_{R}}\right\|=0$.

Proof. Let $H^{2}$ denote the Hardy-Hilbert space with orthonormal basis $\left\{e_{k}: k \geq 0\right\}$. For $k \geq 0$, define $Y: H^{2} \rightarrow \mathcal{F}_{n}$ by $Y e_{k}=U^{k} V \xi$ and $Z: H^{2} \rightarrow \mathcal{F}_{n}$ by $Z e_{k}=U^{k} \nu$ for $k \geq 0$. Note that $Y$ and $Z$ are isometries. For $T$ in $\mathcal{T}_{R}$, by Lemma 3.3 we can factor $T$ as $T=A^{*} B$, for $A$ and $B$ in $\mathcal{L}_{n}$. Then

$$
\begin{aligned}
\left(Y^{*} T Z e_{j}, e_{i}\right) & =\left(A^{*} B U^{j} \nu, U^{i} V \xi\right) \\
& =\left(A^{*} V^{*}\left(U^{*}\right)^{i} U^{j} B \nu, \xi\right) \\
& = \begin{cases}0 & \text { if } i<j \\
c_{i-j} & \text { if } i \geq j\end{cases}
\end{aligned}
$$

where $c_{i-j}=\left(A^{*} B \nu, U^{i-j} V \xi\right)=\left(T \nu, U^{i-j} V \xi\right)$. This implies that $Y^{*} T Z$ is an analytic Toeplitz operator with symbol $f$, for some $f$ in $H^{\infty}$. Note that $\|f\|_{\infty}=$ $\left\|Y^{*} T Z\right\| \leq\|T\|$. Hence

$$
\begin{aligned}
\left|\left(T,\left[\nu \otimes \eta_{k}\right]_{\mathcal{T}_{R}}\right)\right| & =\left|\left(T \nu, \frac{1}{\sqrt{k}} \sum_{i=1}^{k} U^{i} V \xi\right)\right| \\
& =\frac{1}{\sqrt{k}}\left|\sum_{i=1}^{k}\left(T \nu, U^{i} V \xi\right)\right| \\
& =\frac{1}{\sqrt{k}}\left|\sum_{i=1}^{k} c_{i}\right| \\
& \leq \frac{1}{\sqrt{k}}\left\|D_{k}\right\|_{1}\|f\|_{\infty} \\
& \leq \frac{1}{\sqrt{k}}\left\|D_{k}\right\|_{1}\|T\|,
\end{aligned}
$$


where $\left\|D_{k}\right\|_{1}$ denotes the $L^{1}$-norm of the Dirichlet kernel. Using the well-known fact that $\left\|D_{k}\right\|_{1}$ grows logarithmically as $k \rightarrow \infty$ gives $\lim _{k}\left\|\left[\nu \otimes \eta_{k}\right]_{\mathcal{T}_{R}}\right\|=0$.

Lemma 5.4. Given vectors $h_{1}, \ldots, h_{q}$ in $\mathcal{H}$ and $\epsilon>0$, there exists an intertwining operator $Y: \mathcal{F}_{n} \rightarrow \mathcal{H}$ such that $\left\|Y \xi_{\varnothing}\right\|=1$ and $\left\|\left[Y \xi_{\varnothing} \otimes h_{i}\right]_{\mathcal{Z}}\right\|<\epsilon$ for $1 \leq i \leq q$.

Proof. For $1 \leq i \leq q$, let $H_{i}: \mathcal{F}_{n} \rightarrow \mathcal{H}$ be an intertwining operator such that $\left\|H_{i} \xi_{\varnothing}-h_{i}\right\|<\epsilon / 2$. Since $\mathcal{S}$ is type $\mathrm{L}$, by Theorem 4.12 there is an isometric intertwining operator $X: \mathcal{F}_{n} \rightarrow \mathcal{H}$. Then each $H_{i}^{*} X$ is an L-Toeplitz operator, so by Lemma 3.3 we can write $H_{i}^{*} X=A_{i}^{*} B_{i}$, for some $A_{i}$ and $B_{i}$ in $\mathcal{R}_{n}$ such that $A_{i}$ and $B_{i}$ are bounded below. Let $C_{i}=R_{1^{i}{ }_{2}} B_{i}$, and let $D=\sum_{i=1}^{k} R_{1^{i_{2}}} A_{i}$. Then $D$ is bounded below and $H_{i}^{*} X=C_{i}^{*} D$. Using inner-outer factorization, write $D=U F$ for $U$ and $F$ in $\mathcal{R}_{n}$, where $U$ is inner and $F$ is outer. Then $F$ is bounded below since $D$ is, and hence is invertible.

By Lemma 5.2, there exists $m$ such that $\left\|\left(U^{*}\right)^{m} C_{i} \xi_{\varnothing}\right\|<\epsilon /(8\|F\|)$ for $1 \leq i \leq q$. Write $C_{i} \xi_{\varnothing}=\nu_{i}+\omega_{i}$, where $\left\|\omega_{i}\right\|<\epsilon /(8\|F\|)$, and $\nu_{i}$ is in the kernel of $\left(U^{*}\right)^{m}$. Set $V=U^{m} R_{1}$ and $W=U^{m} R_{2}$. Then $V$ and $W$ are isometries in $\mathcal{R}_{n}$ with pairwise orthogonal ranges. Note that $\nu_{i}$ is in the kernel of $V^{*}$. For $k \geq 1$, define intertwining operators $Y_{k}: \mathcal{F}_{n} \rightarrow \mathcal{H}$ by

$$
Y_{k}=X F^{-1} \frac{1}{\sqrt{k+1}} \sum_{j=0}^{k} U^{m-1} R_{1} V^{j} W,
$$

and define

$$
\eta_{k}=\frac{1}{\sqrt{k}} \sum_{j=1}^{k} V^{j} W \xi_{\varnothing} .
$$

Note that $\eta_{k}$ is a unit vector.

Using the fact that $V=D F^{-1} U^{m-1} R_{1}$, we compute

$$
\begin{aligned}
H_{i}^{*} Y_{k} & =H_{i}^{*} X F^{-1} \frac{1}{\sqrt{k+1}} \sum_{j=0}^{k} U^{m-1} R_{1} V^{j} W \\
& =C_{i}^{*} D F^{-1} \frac{1}{\sqrt{k+1}} \sum_{j=0}^{k} U^{m-1} R_{1} V^{j} W \\
& =C_{i}^{*} \frac{1}{\sqrt{k+1}} \sum_{j=1}^{k+1} V^{j} W .
\end{aligned}
$$

Then for $T$ in $\mathcal{T}_{R}$,

$$
\begin{aligned}
\left(T H_{i} \xi_{\varnothing}, Y_{k} \xi_{\varnothing}\right) & =\left(\Phi^{-1}(T) C_{i} \xi_{\varnothing}, \frac{1}{\sqrt{k+1}} \sum_{j=1}^{k+1} V^{j} W \xi_{\varnothing}\right) \\
& =\left(\Phi^{-1}(T) C_{i} \xi_{\varnothing}, \eta_{k+1}\right),
\end{aligned}
$$

Hence $\left\|\left[H_{i} \xi_{\varnothing} \otimes Y_{k} \xi_{\varnothing}\right] \mathcal{Z}\right\|=\left\|\left[C_{i} \xi_{\varnothing} \otimes \eta_{k+1}\right]_{\mathcal{T}_{R}}\right\|$. By Lemma [5.3. we can choose $r$ sufficiently large that $\left\|\left[\nu_{i} \otimes \eta_{r+1}\right]_{\mathcal{T}_{R}}\right\|<\epsilon /(8\|F\|)$. This gives

$$
\begin{aligned}
\left\|\left[C_{i} \xi_{\varnothing} \otimes \eta_{r+1}\right]_{\mathcal{T}_{R}}\right\| & \leq\left\|\left[\nu_{i} \otimes \eta_{r+1}\right]_{\mathcal{T}_{R}}\right\|+\left\|\left[\omega_{i} \otimes \eta_{r+1}\right]_{\mathcal{T}_{R}}\right\| \\
& \left.\leq \|\left[\nu_{i} \otimes \eta_{r+1}\right]_{\mathcal{T}_{R}}\right]\|+\| \omega_{i}\|\| \eta_{r+1} \| \\
& <\epsilon /(4\|F\|) .
\end{aligned}
$$


WANDERING VECTORS AND THE REFLEXIVITY OF FREE SEMIGROUP ALGEBRAS 20

Thus $\left\|\left[H_{i} \xi_{\varnothing} \otimes Y_{k} \xi_{\varnothing}\right]_{\mathcal{Z}}\right\|<\epsilon /(4\|F\|)$ for $1 \leq i \leq q$.

Now,

$$
\begin{aligned}
\left\|Y_{r} \xi_{\varnothing}\right\|^{2} & =\left\|X F^{-1} \frac{1}{\sqrt{r+1}} \sum_{j=0}^{r} U^{m-1} R_{1} V^{j} W \xi_{\varnothing}\right\|^{2} \\
& \geq \frac{1}{(r+1)\|F\|^{2}}\left\|\sum_{j=0}^{r} U^{m-1} R_{1} V^{j} W \xi_{\varnothing}\right\|^{2} \\
& =\frac{r}{(r+1)\|F\|^{2}},
\end{aligned}
$$

which implies

$$
\left\|Y_{r} \xi_{\varnothing}\right\| \geq \frac{1}{2\|F\|} .
$$

Setting $Y=Y_{r} /\left\|Y_{r} \xi_{\varnothing}\right\|$, it follows that

$$
\begin{aligned}
\left\|\left[H_{i} \xi_{\varnothing} \otimes Y \xi_{\varnothing}\right]_{\mathcal{Z}}\right\| & =\frac{1}{\left\|Y_{p} \xi_{\varnothing}\right\|}\left\|\left[H_{i} \xi_{\varnothing} \otimes Y_{p} \xi_{\varnothing}\right]_{Z}\right\| \\
& \leq 2\|F\|\left\|\left[H_{i} \xi_{\varnothing} \otimes Y_{k} \xi_{\varnothing}\right]_{\mathcal{Z}}\right\| \\
& <\epsilon / 2 .
\end{aligned}
$$

Thus

$$
\begin{aligned}
\left\|\left[h_{j} \xi_{\varnothing} \otimes Y \xi_{\varnothing}\right]_{Z}\right\| & \leq\left\|\left[\left(h_{j}-H_{j} \xi_{\varnothing}\right) \otimes Y \xi_{\varnothing}\right]_{\mathcal{Z}}\right\|+\left\|\left[H_{j} \xi_{\varnothing} \otimes Y \xi_{\varnothing}\right]_{\mathcal{Z}}\right\| \\
& \leq\left\|h_{j}-H_{j} \xi_{\varnothing}\right\|\left\|Y \xi_{\varnothing}\right\|+\left\|\left[H_{j} \xi_{\varnothing} \otimes Y \xi_{\varnothing}\right]_{\mathcal{Z}}\right\| \\
& <\epsilon .
\end{aligned}
$$

Lemma 5.5. Given vectors $h_{1}, \ldots, h_{q}$ in $\mathcal{H}, p \geq 1$, and $\epsilon>0$, there exists a unit vector $z$ in $\mathcal{H}$ such that

$$
\left\|\left[S_{u_{1}} z \otimes S_{u_{2}} z\right]_{\mathcal{S}}-\phi\left(\left[\xi_{u_{1}} \otimes \xi_{u_{2}}\right]_{\mathcal{L}_{n}}\right)\right\|<\epsilon
$$

for all $u_{1}$ and $u_{2}$ in $\mathbb{F}_{n}^{p}$, and such that $\left\|\left[S_{w} z \otimes h_{i}\right]_{\mathcal{S}}\right\|<\epsilon$ and $\left\|\left[h_{i} \otimes S_{w} z\right]_{\mathcal{S}}\right\|<\epsilon$ for all $w \in \mathbb{F}_{n}^{+}$and $1 \leq i \leq q$.

Proof. By Lemma 5.4, there is an intertwining operator $Y: \mathcal{F}_{n} \rightarrow \mathcal{H}$ such that $\left\|Y \xi_{\varnothing}\right\|=1$ and $\left\|\left[Y \xi_{\varnothing} \otimes h_{i}\right]_{\mathcal{Z}}\right\|<\epsilon$ for $1 \leq i \leq q$. By Lemma 4.6, there is a word $v$ in $\mathbb{F}_{n}^{+}$such that $\left\|\left[S_{u_{1} v} Y \xi_{\varnothing} \otimes S_{u_{2} v} Y \xi_{\varnothing}\right]_{\mathcal{S}}-\phi\left(\left[\xi_{u_{1}} \otimes \xi_{u_{2}}\right]_{\mathcal{L}_{n}}\right)\right\|<\epsilon$ for all words $u_{1}$ and $u_{2}$ in $\mathbb{F}_{n}^{p}$. Set $z=S_{v} Y \xi_{\varnothing}$.

For $T$ in $\mathcal{Z}$ and $w \in \mathbb{F}_{n}^{+}$,

$$
\begin{aligned}
\left|\left(T,\left[S_{w} z \otimes h_{j}\right]_{Z}\right)\right| & =\left|\left(T S_{w},\left[z \otimes h_{j}\right]_{\mathcal{Z}}\right)\right| \\
& \leq\left\|T S_{w}\right\|\left\|\left[z \otimes h_{j}\right]_{\mathcal{Z}}\right\| \\
& \leq\|T\|\left\|\left[z \otimes h_{J}\right]_{\mathcal{Z}}\right\| .
\end{aligned}
$$

Hence $\left\|\left[S_{w} z \otimes h_{i}\right]_{\mathcal{Z}}\right\| \leq\left\|\left[z \otimes h_{i}\right]_{\mathcal{Z}}\right\|<\epsilon$ and similarly, $\left\|\left[h_{i} \otimes S_{w} z\right]_{Z}\right\| \leq\left\|\left[h_{i} \otimes z\right]_{\mathcal{Z}}\right\|<\epsilon$. In particular, restricting to $\mathcal{S}$ gives $\left\|\left[S_{w} z \otimes h_{i}\right]_{\mathcal{S}}\right\|<\epsilon$ and $\left\|\left[h_{i} \otimes S_{w} z\right]_{\mathcal{S}}\right\|<\epsilon$.

Lemma 5.5 is essentially a strengthened version of Lemma 4.9, in the sense that the $h_{i}$ 's in the hypothesis can be completely arbitrary.

Lemma 5.6. Given a weak ${ }^{*}$-continuous linear functional $\pi$ on $\mathcal{S}, h_{1}, \ldots, h_{q}$ in $\mathcal{H}$, and $\epsilon>0$, there are vectors $x$ and $y$ in $\mathcal{H}$ such that 
(1) $\left\|\pi-[x \otimes y]_{\mathcal{S}}\right\|<\epsilon$,

(2) $\|x\|<(1+\epsilon)\|\pi\|^{1 / 2}$ and $\|y\|<(1+\epsilon)\|\pi\|^{1 / 2}$,

(3) $\left\|\left[x \otimes h_{j}\right]_{\mathcal{S}}\right\|<\epsilon$ and $\left\|\left[h_{j} \otimes y\right]_{\mathcal{S}}\right\|<\epsilon$ for $1 \leq j \leq q$.

Proof. The proof follows exactly as in the proof of Lemma 4.11, using Lemma 5.5 in place of Lemma 4.10 .

Lemma 5.6 clearly implies the desired result.

Theorem 5.7. Every type $L$ free semigroup algebra with $n \geq 2$ generators has property $\mathcal{X}_{0,1}$.

Corollary 5.8. Every type $L$ free semigroup algebra with $n \geq 2$ generators is hyper-reflexive with hyper-reflexivity constant at most 3.

Remark 5.9. We note here that the results in Section 5 can be proved independently of Section 4 Let $\mathcal{S}$ be a type L free semigroup algebra. By Lemma 2.1, for some $m$, the ampliation $\mathcal{S}^{(m)}$ has a wandering vector. By the results in this section, $\mathcal{S}^{(m)}$ has property $\mathcal{X}_{0,1}$. By Theorem 3.8 of [5], it follows that $\mathcal{S}$ has property $\mathcal{X}_{(m-1 / m), 1}$, which by [4] implies that $\mathcal{S}$ is hyper-reflexive, and in particular is reflexive. It follows that $\mathcal{S}$ has a wandering vector, and we can apply the results of Section 5 to obtain that $\mathcal{S}$ has property $\mathcal{X}_{0,1}$.

\section{Concluding Remarks}

In [10], Davidson, Katsoulis, and Pitts posed the following four questions about free semigroup algebras.

(1) Can a free semigroup algebra be a von Neumann algebra?

(2) Does every type L free semigroup algebra have a wandering vector?

(3) Is every free semigroup algebra reflexive, or even hyper-reflexive?

(4) Is the restriction of a type $L$ free semigroup algebra to an invariant subspace also of type L?

In [21] (see also [9]), Read answered (1) in the affirmative by showing that $\mathcal{B}(\mathcal{H})$ is a free semigroup algebra. Theorem 4.12 gives an affirmative answer to (2), and Corollary 4.14 gives an affirmative answer to the first part of (3). Corollary 4.15 partially answers the second part of (3) in the affirmative, as we now explain.

Recall that by the general structure theorem for free semigroup algebras [10], every free semigroup algebra decomposes into $2 \times 2$ block-lower-triangular form, where the left column is a slice of a von Neumann algebra, and the bottom right entry is a type L free semigroup algebra. By Corollary 4.15, we know that the type L part is hyper-reflexive. The difficulty in proving the hyper-reflexivity of the entire algebra comes down to the fact that it is an open question whether every von Neumann algebra is hyper-reflexive.

For $n=1$, (4) has a negative answer, as the following example from [10] shows.

Example 6.1. Let $U$ denote the bilateral shift, i.e. the operator of multiplication by $z$ on $L^{2}(\mathbb{T}, \mu)$, where $\mu$ is Lebesgue measure. Let $\mathbf{1}_{[0, \pi]}$ and $\mathbf{1}_{[\pi, 2 \pi]}$ denote the characteristic function for the intervals $[0, \pi]$ and $[\pi, 2 \pi]$ respectively, and define measures $\mu_{1}$ and $\mu_{2}$ by $\mu_{1}=\mathbf{1}_{[0, \pi]} \mu$ and $\mu_{2}=\mathbf{1}_{[\pi, 2 \pi]} \mu$. Let $U_{1}$ and $U_{2}$ be the operators of multiplication by $z$ on $L^{2}\left(\mathbb{T}, \mu_{1}\right)$ and $L^{2}\left(\mathbb{T}, \mu_{2}\right)$ respectively. Since the support of $\mu_{1}$ and $\mu_{2}$ are proper measurable subsets of the circle, by [22], the weakoperator-closed algebras generated by $U_{1}$ and $U_{2}$ are self-adjoint. On the other 
hand, $U_{1} \oplus U_{2}$ is unitarily equivalent to the bilateral shift $U$, so the weak-operatorclosed algebra it generates is isomorphic to the analytic Toeplitz algebra.

For $n \geq 2$, the answer to (4) is unknown, but it is related to the notion of absolute continuity, which we now discuss.

The non-commutative analytic disk algebra $\mathcal{A}_{n}$ is the norm-closed (non-selfadjoint) algebra generated by $L_{1}, \ldots, L_{n}$. Popescu [19] showed that the norm-closed algebra generated by any row isometry of size $n$ is completely isometrically isomorphic to $\mathcal{A}_{n}$.

Definition 6.2. Let $\sigma$ be a $*$-extendible representation of $\mathcal{A}_{n}$ on a Hilbert space $\mathcal{H}$. These are precisely the representations with the property that $\left[\begin{array}{llll}\sigma\left(S_{1}\right) & \cdots & \sigma\left(S_{n}\right)\end{array}\right]$ is a row isometry. We say that $\sigma$ is absolutely continuous if, for every $x$ in $\mathcal{H}$, the linear functional on $\mathcal{A}_{n}$ given by

$$
A \rightarrow(\sigma(A) x, x), \quad A \in \mathcal{A}_{n}
$$

extends to a weak*-continuous linear functional on $\mathcal{L}_{n}$.

If a representation $\sigma$ is type $\mathrm{L}$, i.e. if $\sigma\left(L_{1}\right), \ldots, \sigma\left(L_{n}\right)$ generate a type $\mathrm{L}$ free semigroup algebra, then the fact that every type $\mathrm{L}$ free semigroup algebra is completely isometrically isomorphic and weak*-to-weak* homeomorphic to $\mathcal{L}_{n}$ immediately implies that $\sigma$ is absolutely continuous. In [11, Davidson, Li, and Pitts conjectured that for $n \geq 2$, the converse of this holds. In other words, they conjectured that for $n \geq 2$, every absolutely continuous representation of $\mathcal{A}_{n}$ is actually of type L.

For $n=1$, this is not true. Indeed, it's clear from Definition 6.2 that a representation which is a direct summand of an absolutely continuous representation is also absolutely continuous. It follows that $U_{1}$ and $U_{2}$ from Example 6.1 correspond to absolutely continuous representations. However, the weak-operator-closed algebras generated by $U_{1}$ and $U_{2}$ are self-adjoint, and in particular are not of type $\mathrm{L}$.

A proof of the conjecture would therefore provide an interesting example of a result in the non-commutative setting which has no commutative counterpart. On the other hand, it would be even more interesting if this conjecture was false, as we now explain.

It was shown in [13] that for $n \geq 2$, if $\sigma$ is an absolutely continuous representation of $\mathcal{A}_{n}$, then the infinite ampliation $\sigma^{(\infty)}$ is a representation of type $\mathrm{L}$. In this case, the weak*-closed algebra generated by $\sigma\left(L_{1}\right), \ldots, \sigma\left(L_{n}\right)$ is algebraically isomorphic to $\mathcal{L}_{n}$, and so is non-self-adjoint, while the weak-operator-closed algebra generated by $\sigma\left(L_{1}\right), \ldots, \sigma\left(L_{n}\right)$ is self-adjoint. This is not completely implausible, as Loebl and Muhly 15 have demonstrated the existence of weak*-closed non-self-adjoint algebras of analytic type whose weak closure is self-adjoint.

Our results seem to provide some evidence that this conjecture is true. Davidson, $\mathrm{Li}$, and Pitts [1] showed that if an absolutely continuous representation of $\mathcal{A}_{n}$ has a wandering vector, then it is type L. On the other hand, we know by Theorem 4.12 that if a representation of $\mathcal{A}_{n}$ is type $\mathrm{L}$, then it has a wandering vector. Therefore, the truth of the conjecture is equivalent to the existence of a wandering vector for every absolutely continuous representation.

For $n \geq 2$, suppose that $\sigma$ is an absolutely continuous representation of $\mathcal{A}_{n}$. One would like to use the methods in the present paper to find a wandering vector for $\sigma$. Unfortunately, we no longer know that some finite ampliation of $\sigma$ is type L, which means we can't make use of Theorem 2.1. However, most of our approximation 
techniques do still work in this setting, so it seems plausible that some modification of our methods which avoids the dependence on Theorem 2.1 could yield a wandering vector for $\sigma$.

Acknowledgement. The author is grateful to Ken Davidson for his advice and support.

\section{REFERENCES}

1. William Arveson, Interpolation problems in nest algebras, J. Funct. Anal. 20 (1975), no. 3, 208-233. MR MR0383098 (52 \#3979)

2. William B. Arveson, Subalgebras of $C^{*}$-algebras, Acta Math. 123 (1969), 141-224. MR MR0253059 (40 \#6274)

3. Hari Bercovici, Factorization theorems and the structure of operators on Hilbert space, Ann. Math. (2) 128 (1988), no. 2, 399-413. MR MR960951 (89i:47032)

4. - Hyper-reflexivity and the factorization of linear functionals, J. Funct. Anal. 158 (1998), no. 1, 242-252. MR MR1641578 (99g:47105)

5. Hari Bercovici, Ciprian Foias, and Carl Pearcy, Dual algebras with applications to invariant subspaces and dilation theory, CBMS Regional Conference Series in Mathematics, vol. 56, Published for the Conference Board of the Mathematical Sciences, Washington, DC, 1985. MR MR787041 (87g:47091)

6. Scott W. Brown, Some invariant subspaces for subnormal operators, Integral Equations Operator Theory 1 (1978), no. 3, 310-333. MR MR511974 (80c:47007)

7. Man Duen Choi, A Schwarz inequality for positive linear maps on $C^{*}$-algebras, Illinois J. Math. 18 (1974), 565-574. MR MR0355615 (50 \#8089)

8. Kenneth R. Davidson, The distance to the analytic Toeplitz operators, Illinois J. Math. 31 (1987), no. 2, 265-273. MR MR882114 (88e:47050)

9. $-\mathcal{B}(\mathcal{H})$ is a free semigroup algebra, Proc. Amer. Math. Soc. 134 (2006), no. 6, 17531757 (electronic). MR MR2204288 (2007b:47190)

10. Kenneth R. Davidson, Elias Katsoulis, and David R. Pitts, The structure of free semigroup algebras, J. Reine Angew. Math. 533 (2001), 99-125. MR MR1823866 (2002a:47107)

11. Kenneth R. Davidson, Jiankui Li, and David R. Pitts, Absolutely continuous representations and a Kaplansky density theorem for free semigroup algebras, J. Funct. Anal. 224 (2005), no. 1, 160-191. MR MR2139108 (2006f:47088)

12. Kenneth R. Davidson and David R. Pitts, Invariant subspaces and hyper-reflexivity for free semigroup algebras, Proc. London Math. Soc. (3) 78 (1999), no. 2, 401-430. MR MR1665248 (2000k:47005)

13. Kenneth R. Davidson and Dilian Yang, A note on absolute continuity in free semigroup algebras, Houston J. Math. 34 (2008), no. 1, 283-288. MR MR2383708 (2009c:47122)

14. David W. Kribs, Factoring in non-commutative analytic Toeplitz algebras, J. Operator Theory 45 (2001), no. 1, 175-193. MR MR1823067 (2002g:47151)

15. Richard I. Loebl and Paul S. Muhly, Analyticity and flows in von Neumann algebras, J. Funct. Anal. 29 (1978), no. 2, 214-252. MR MR504460 (81h:46080)

16. A. N. Loginov and V. S. Šul'man, Hereditary and intermediate reflexivity of $W^{*}$-algebras, Izv. Akad. Nauk SSSR Ser. Mat. 39 (1975), no. 6, 1260-1273, 1437. MR MR0405124 (53 \#8919)

17. Gelu Popescu, Multi-analytic operators and some factorization theorems, Indiana Univ. Math. J. 38 (1989), no. 3, 693-710. MR MR1017331 (90k:47019)

18. _ von Neumann inequality for $\left(B(\mathcal{H})^{n}\right)_{1}$, Math. Scand. 68 (1991), no. 2, 292-304. MR MR1129595 (92k:47073)

19. , Non-commutative disc algebras and their representations, Proc. Amer. Math. Soc. 124 (1996), no. 7, 2137-2148. MR MR1343719 (96k:47077)

20. - Noncommutative transforms and free pluriharmonic functions, Adv. Math. 220 (2009), no. 3, 831-893. MR MR2483229

21. Charles J. Read, A large weak operator closure for the algebra generated by two isometries, J. Operator Theory 54 (2005), no. 2, 305-316. MR MR2186356 (2006g:47119)

22. John Wermer, On invariant subspaces of normal operators, Proc. Amer. Math. Soc. 3 (1952), 270-277. MR MR0048700 (14,55g) 
WANDERING VECTORS AND THE REFLEXIVITY OF FREE SEMIGROUP ALGEBRAS 24

Pure Math. Dept., University of Waterloo, Waterloo, Ontario, Canada N2L 3G1

E-mail address: m3kennedy@uwaterloo.ca 